\title{
On the Lorentz structure of the symmetry energy
}

\author{
T. Gaitanos ${ }^{\mathrm{a}}$, M. Di Toro ${ }^{\mathrm{a}, 1}, \mathrm{~S}$. Type $\mathrm{l}^{\mathrm{b}}$, V. Baran ${ }^{\mathrm{a}, 2}$, \\ C. Fuchs ${ }^{\mathrm{c}}$, V. Greco ${ }^{\mathrm{d}, 3}$ and H.H. Wolter ${ }^{\mathrm{e}}$ \\ ${ }^{a}$ Laboratori Nazionali del Sud INFN, I-95123 Catania, Italy \\ Physics 85 Astronomy Dept., Univ. of Catania \\ ${ }^{\mathrm{b}}$ Gesellschaft für Schwerionenforschung, D-64291 Darmstadt, Germany \\ ${ }^{\mathrm{c}}$ Institute für Theoretische Physik, Universität Tübingen, D-72076 Tübingen, \\ Germany \\ ${ }^{\mathrm{d}}$ Cyclotron Institute, Texas A\& M University, College Station, USA \\ e Sektion Physik, Universität München, D-85748 Garching, Germany
}

\begin{abstract}
We investigate in detail the density dependence of the symmetry energy in a relativistic description by decomposing the iso-vector mean field into contributions with different Lorentz covariant properties. We find important effects of the iso-vector, scalar channel (i.e. $\delta$-meson like) on the high density behavior of the symmetry energy. Applications to static properties of finite nuclei and to dynamic situations of heavy ion collisions are explored and related to each other. The nuclear structure studies show only moderate effects originating from the virtual $\delta$ meson. At variance, in heavy ion collisions one finds important contributions on the reaction dynamics arising from the different Lorentz structure of the high density symmetry energy when a scalar iso-vector $\delta$ field is introduced. Particularly interesting is the related neutron/proton effective mass splitting for nucleon transport effects and for resonance and particle production around the threshold. We show that the $\delta$-like channel turns out to be essential for the production of pions, when comparing with experimental data, in particular for high momentum selections.
\end{abstract}

Key words: Asymmetric nuclear matter, symmetry energy, finite nuclei, relativistic heavy ion collisions, particle production.

PACS numbers: 25.75.-q, 21.30.Fe, 21.65+f, 25.60-t

$\overline{1}$ ditoro@lns.infn.it

2 On leave from NIPNE-HH and Bucharest University, Romania

3 Supported by a INFN Post-Doc Fellowship

Preprint submitted to Elsevier Preprint

16 October 2018 


\section{Introduction}

Heavy ion collisions at relativistic energies from 0.1 up to $1-2 \mathrm{AGeV}$ offer the possibility to access the equation of state $(E O S)$ of nuclear matter under extreme conditions of density, isospin and temperature [1]. This knowledge is important in understanding many interesting astrophysical phenomena such as the physical mechanism of supernovae explosions and the neutron star structure and cooling.

During the last three decades one has attempted in many ways to investigate the properties of highly excited hadronic matter. In phenomenological models of a relativistic quantum field theory, like the Relativistic Mean Field $(R M F)$ model of Quantum-Hadro-Dynamics $(Q H D)[2]$, a significant decrease of the effective nucleon mass with density was found to be essential for a reliable description of infinite nuclear matter and finite nuclei. More microscopic studies, the so-called Dirac-Brueckner-Hartree-Fock ( $D B H F)$ approach, based on realistic nucleon-nucleon $(N N)$ interactions in the One-Boson-Exchange $(O B E)$ model, see refs.[3-6], also predict a strong decrease of the effective nucleon mass. Similar effects have been found for particles with strangeness such as kaons [7-9]. The results can be represented by saying that the effective nucleonmeson vertices or coupling functions exhibit a density dependence and, for energies higher than the Fermi energy, an additional momentum dependence [6]. As a consequence the EOS predictions of different models show quite variable density dependences for densities higher than saturation, characterized by referring to a hard or soft $E O S$, which have been tested in heavy ion collisions $[10]$.

So far asymmetric nuclear matter has been only poorly investigated, but certainly the same considerations appear for the density dependence of the symmetry term which are directly related to the Lorentz structure of the effective fields in the isovector channel and to their weights (the coupling functions) in different baryon density regions. Around the saturation point the $a_{4}$ parameter of the Weizsäecker mass formula contains the leading information (in a sense equivalent to the $a_{1}$ parameter for the isoscalar part). Actually, there is quite a wide range of extracted values, roughly from 28 to $36 \mathrm{MeV}$, depending on the way the surface effects are accounted for. This clearly shows that even to reproduce the equilibrium EOS point we need a better knowledge of the density dependence of the symmetry term since the slope (the symmetry pressure) and the curvature (the symmetry compressibility) around $\rho_{0}$ are also playing an important role in reproducing nuclear masses. A related sensitive observable appears to be the difference between neutron and proton r.m.s. radii in charge asymmetric nuclei. The wide range of predictions made by different relativistic (and non-relativistic Skyrme- $H F$ ) effective interactions has been nicely presented recently in the refs. $[11,12]$. Other independent information on 
the symmetry energy in dilute matter, below saturation, have been suggested from heavy ion collisions in the Fermi energy domain, in particular from the isospin distillation mechanism in fragment production, [13] and refs. therein, and from the isospin content of fast particle emissions, [14].

In standard $R M F$ models the iso-vector part of the mean field is described in terms of a constant vector, isovector, $\rho$-coupling giving an almost linear density dependence of the symmetry energy. Recently the importance of a contribution of the scalar isovector channel, the virtual $\delta\left[a_{0}(980)\right]$ meson, within the same $R M F$ framework, has been stressed [15,16]. This mostly affects the high density behavior, as we will show in the following. We note that the $\delta$-channel usually has not been considered before just on the basis of the weak contribution to the free Nucleon-Nucleon interaction. But in the spirit of the Effective Field Theory as a relativistic Density Functional Theory, (the EFT/DFT framework, see $[17,18]$ ), the relevance of this channel could be completely different in nuclear matter, due to medium and many-body effects. We like to note that very recently, see the conclusions of ref.[12], the $\delta$-channel has been reconsidered as an interesting improvement of covariant approaches, in the framework of the EFT/DFT philosophy. One of the main tasks of our work is just to try to select the dynamical observables more sensitive to it.

In the Density Dependent Hadronic ( $D D H$ ) field theory [19], the detailed density dependence of the nucleon-meson couplings has been taken either directly from $D B H F$ calculations [20-22] or has been fitted to data of nuclear matter and finite nuclei [23]. Certainly the correct microscopic approach should be to derive the coupling functions, in a $Q H D$ mean field scheme, from DiracBrueckner-Hartree-Fock calculations. Several attempts have been performed so far, see refs. [20-22], but the results do not agree and appear to be model dependent, in particular for the isovector channel. We will discuss such point in more detail below.

Aim of the present work is to try to shed some light on this intricate puzzle of the baryon density dependence of the symmetry term, in particular in the high density region. For densities beyond saturation one needs extrapolations which can only be investigated in dynamical situations of heavy ion collisions at intermediate energies, where hot and dense asymmetric nuclear matter is formed. Indeed, the study of the high density symmetry energy is an object of recently started investigations [24-27]. Their motivation has been the planning of new experimental heavy ion facilities with neutron-rich radioactive beams with data available in the near future.

Here we will actually explore the properties of asymmetric nuclear matter in different nuclear systems, i.e. finite nuclei and heavy ion collisions. For our studies we apply various versions of $Q H D$ models, in the Relativistic Mean Field $(R M F)$ approach, which differ in the treatment of the effec- 
tive meson-nucleon vertices. In the standard $R M F$ models the nucleon-meson couplings are given by constants fitted to nuclear matter saturation properties. Some non-linear contributions for the meson fields are considered, $N L-$ $R M F$ approaches, which imply definite density dependences in the model. The $R M F-D D H$ theory uses the so-called Vector Density Dependent $(V D D)$ parametrizations $[19,23]$ in terms of directly density dependent effective coupling functions. In this case there is no need of non-linear terms.

In this context relativity is important because it naturally explains essential nuclear matter properties such as the cancellation of large Lorentz vector and scalar fields, in the isoscalar channel, leading to a small value of $16 \mathrm{MeV}$ for the binding energy per nucleon and, simultaneously, to a strong spin-orbit potential. Within a covariant formulation one is also able to better understand the origin of the repulsive and attractive contributions to the effective $N N$ interaction. The separation of the total nuclear mean field potential in terms of its Lorentz components is of particular importance when studying dynamical situations of heavy ion collisions at relativistic energies [25,28,29].

In the $R M F$ picture the nuclear mean field is given by the exchange of mesons with different Lorentz properties. The iso-scalar part which describes the EOS of symmetric nuclear matter is mainly characterized by an attractive scalar field ( $\sigma$-meson) and repulsive vector field ( $\omega^{\mu}$-meson) both with contributions of the same order $400-500 \mathrm{MeV}$ to the total energy per nucleon. The corresponding masses, fixed as $m_{\sigma} \sim 550 \mathrm{MeV}$ and $m_{\omega}=783 \mathrm{MeV}$, are important in finite nuclei calculations. One should note the importance of both these mesons in describing the saturation properties of symmetric nuclear matter.

A covariant treatment of the problem is also helpful for the understanding of the properties of asymmetric nuclear and neutron matter. As in the iso-scalar case, the iso-vector part of the nuclear mean field, which is responsible for the density dependence of the symmetry energy, can again be characterized by iso-vector mesons with different Lorentz properties. These are the iso-vector, vector field ( $\vec{\rho}^{\mu}$-meson) and the iso-vector, scalar field $(\vec{\delta}$-meson $)$, with masses $m_{\rho}=769 \mathrm{MeV}$ (763 MeV in $V D D$ models $)$ and $m_{\delta}=980 \mathrm{MeV}$. Due to less available experimental information several possibilities have been proposed to describe the density dependence of the symmetry energy.

In contrast to the iso-scalar case, since here we do not have the stringent saturation condition of balancing attractive (scalar) and repulsive (vector) contributions, one can apply either only the $\rho$-meson, or both, the $\rho$ - and $\delta$ mesons. Because of the different Lorentz properties of the mesons one expects different density behavior of the symmetry energy especially at high densities. The argument is very simple and clearly discussed in the refs. $[15,16]$. Vector and scalar effective fields will contribute to the symmetry term with quantities proportional to baryon and scalar densities, respectively. As a consequence the 
main differences should appear at baryon densities above saturation. Of course such direct interplay between $\rho$ and $\delta$ contributions to the symmetry energy can be reproduced in the symmetry term just by introducing density dependent isovector/vector couplings without the need of a new virtual (scalar) meson in the isospin channel. The scalar nature of the $\delta$-meson, however, also leads to a new effect, the splitting of the neutron and proton effective masses, with interesting consequences on nucleon transport properties and on particle and resonance production in the reaction dynamics at high baryon and isospin densities. In this work we will focus our attention on these new features of a isovector/scalar coupling in realistic collision simulations and on the related most sensitive experimental observables. Charged pion yields appear to be a suitable quantity to measure in order to see such effects.

As we shall show, finite nuclei studies cannot separate easily between the effects originating from the different iso-vector mesons for densities mainly below saturation, see also [23]. Therefore, relativistic heavy ion collisions represent a unique tool to study the properties of highly compressed asymmetric nuclear matter using radioactive beams.

The paper is organized as follows. In Section 2 we discuss asymmetric nuclear matter within the $R M F$ theory. The models are then applied to static cases of finite nuclei (Sec.3) and to dynamical ones of heavy ion collisions (Secs.4, $5)$. The application to finite nuclei is important to demonstrate the reliability of the models in this sector, and the role of the $\delta$-meson will be particularly emphazised. Then we describe briefly the theoretical treatment and numerical procedure of heavy ion collisions. The transport theoretical analysis is mainly applied here to particle production. In particular we focus the attention on charged pion yields, with rapidity and transverse momentum selections (Sec.5). A comparison with experiments is performed, when data are available, also in a preliminary status. Final remarks and an outlook follow in the last Section.

\section{Equation of state of asymmetric nuclear matter}

The starting point of a covariant quantum field theory is the Lagrangian of an interacting many body system of baryons and mesons. The latter determines the interaction between baryons in terms of nucleon-meson coupling vertices. Within a Non-Linear Relativistic Mean Field $(N L-R M F)$ approximation the baryons are given by Dirac spinors and the mesons are described by classical field equations

$$
\left[\gamma_{\mu} i \partial^{\mu}-g_{\omega} \omega_{0} \gamma^{0}-g_{\rho} \gamma^{0} \tau_{3} \rho_{0}-\left(M-g_{\sigma} \sigma-g_{\delta} \tau_{3} \delta_{3}\right)\right] \Psi=0
$$




\begin{tabular}{|l|c|c|c|c|c|c|c|}
\hline \hline & $f_{\sigma}\left(f m^{2}\right)$ & $f_{\omega}\left(f m^{2}\right)$ & $f_{\rho}\left(f m^{2}\right)$ & $f_{\delta}\left(f m^{2}\right)$ & $\mathrm{A}\left(f m^{-1}\right)$ & $\mathrm{B}$ & \\
\hline \hline$N L \rho$ & 15.60 & 10.50 & 1.10 & 0.0 & 0.015 & -0.004 & \\
\hline$N L \rho \delta$ & 15.60 & 10.50 & 3.15 & 2.4 & 0.015 & -0.004 & \\
\hline$N L 3$ & 15.73 & 10.53 & 1.34 & 0.0 & 0.01 & -0.003 & \\
\hline \hline
\end{tabular}

Table 1

Coupling parameters in terms of $f_{i} \equiv\left(\frac{g_{i}}{m_{i}}\right)^{2}$ for $i=\sigma, \omega, \rho, \delta, A \equiv \frac{a}{g_{\sigma}^{3}}$ and $B \equiv \frac{b}{g_{\sigma}^{4}}$ for the non-linear RMF models using the $\rho$ and both, the $\rho$ and $\delta$ mesons for the characterization of the isovector mean field in comparison with the NL3 model. See text.

$$
\begin{aligned}
& m_{\sigma}^{2} \sigma+a \sigma^{2}+b \sigma^{3}=g_{\sigma}<\hat{\bar{\Psi}} \hat{\Psi}>=g_{\sigma} \rho_{s} \\
& m_{\omega}^{2} \omega^{\mu}=g_{\omega}<\hat{\bar{\Psi}} \gamma^{\mu} \hat{\Psi}>=g_{\omega} j^{\mu} \\
& m_{\rho}^{2} \rho^{0}=g_{\rho}<\hat{\bar{\Psi}} \gamma^{0} \tau_{3} \hat{\Psi}>=g_{\rho} \rho_{3} \\
& m_{\delta}^{2} \delta_{3}=g_{\delta}<\hat{\bar{\Psi}} \tau_{3} \hat{\Psi}>=g_{\delta} \rho_{s 3} .
\end{aligned}
$$

The nuclear system is characterized by a baryonic quantum field and a surrounding background of classical mesonic fields, the iso-scalar, scalar $\sigma$-, the iso-scalar, vector $\omega$-, the iso-vector, scalar $\delta$ - and the iso-vector, vector $\rho$ mesons. The isospin vector and scalar densities are given by $\rho_{3}=\rho_{p}-\rho_{n}, \rho_{s 3}=$ $\rho_{s p}-\rho_{s n}$, respectively, $\rho_{p, n}$ being the proton and neutron densities. The isoscalar $\sigma$ field contains non-linear contributions. In $D D H-R M F$ approaches the above equations will be modified for the presence of rearrangement terms in the nucleon self-energies and for the lack on non-linear terms, see refs. $[19,23]$.

The energy-momentum tensor within the same non-linear $R M F$ approach is given by $\left(f_{i} \equiv\left(g_{i} / m_{i}\right)^{2}\right)$ :

$$
\begin{aligned}
T^{\mu \nu}(x) & =t^{\mu \nu}+f_{\omega} j^{\mu} j^{\nu}+f_{\rho} j_{3}^{\mu} j_{3}^{\nu} \\
& +\frac{1}{2} g^{\mu \nu}\left[m_{\sigma} \sigma^{2}+2 U(\sigma)+f_{\delta} \rho_{s 3}^{2}-f_{\omega} j^{\alpha} j_{\alpha}-f_{\rho} j_{3}^{\alpha} j_{3 \alpha}\right]
\end{aligned}
$$

with $t^{\mu \nu}$ the kinetic part, $t^{\mu \nu}(x) \equiv i<\hat{\bar{\Psi}} \gamma^{\mu} \partial^{\nu} \hat{\Psi}>$, and $U(\sigma)$ the non-linear contributions of the iso-scalar $\sigma$-meson $U \equiv \frac{1}{3} a \sigma^{3}+\frac{1}{4} b \sigma^{4}$. The effective mass is now different for protons and neutrons due to the appearance of the iso-vector, scalar $\delta$-meson [30]:

$$
\left.m_{i}^{*}=M-g_{\sigma} \sigma \pm g_{\delta} \delta_{3} \quad \text { (- proton, }+ \text { neutron }\right) \quad \text {. }
$$

For the investigation of asymmetric nuclear matter and neutron matter we 
introduce the asymmetry parameter $\alpha=\frac{\rho_{n}-\rho_{p}}{\rho_{n}+\rho_{p}}$ which describes the relative ratio of the neutron to proton fraction of the nuclear matter. The asymmetric nuclear matter calculations can be done by solving selfconsistently the Eqs. (1-5,7). The parameters of the model, fixed to nuclear matter saturation properties, are given in the Table 1 and compared to the widely used NL3 parametrization $[31,32]$. In order to isolate the effects in the isovector channel we have chosen on purpose very similar parameters in the isoscalar part. At saturation in symmetric matter we have a density $\rho_{\text {sat }}=0.148 \mathrm{fm}^{-3}$, a nucleon effective mass $m_{N}^{*}=0.6 M$ and a compressibility $K_{N M}=271.7 \mathrm{MeV}$. In $N L 3$ the symmetry parameter is $a_{4}=37.4 M e V$ while in our $N L \rho, N L \rho \delta$ choices we fit a value $a_{4}=30.5 \mathrm{MeV}$.

In all the above $N L-R M F$ schemes the nucleon-meson couplings are given by constant values, see Table 1 . We also want to compare to $R M F$ models based on the Density Dependent Hadronic $(D D H)$ field approach [19], since they have been shown to reproduce well nuclear matter and finite nuclei $[20,23,33]$. We will follow here in particular the parametrization of ref.[23], where the density dependence has been fitted to finite nuclei, and which makes a prediction for the isovector channel at high density. The symmetry energy at saturation is chosen as $a_{4} \sim 33.4 \mathrm{MeV}$, see also ref.[33]. The corresponding properties for symmetric matter are: density $\rho_{\text {sat }}=0.153 \mathrm{fm}^{-3}$, nucleon effective mass $m_{N}^{*}=0.55 M$ and compressibility $K_{N M}=240.0 \mathrm{MeV}$. So the interactions with density dependent couplings used in this work are softer with respect to the $N L-R M F$ models. We will see that this will affect the particle production yields.

However, the work of ref.[23] made no predictions for the $\delta$-like field, since there was not much sensitivity to it in finite nuclei, as expected (see Sect.1) and as we will also see here later in Sect.3. To test this part in heavy ion collisions we use three variants of these $D D H$ models. $D D H \rho$ is just the $V D D$ parametrization of ref.[23], with one a $\rho$-like isovector field with an exponential density dependence of the coupling. In the models $D D H 3$ we use the functional form of the vertex density dependences for the $\rho$ - and $\delta$-fields as extracted from the $D B H F$ calculations of ref.[34]. In $D D H 3 \rho$ we use only the $\rho$-field and normalize to the $a_{4} \sim 33.4 \mathrm{MeV}$ value at saturation, as in the $D D H \rho(V D D)$ case. In the $D D H \rho \delta$ we use $\rho$ - and $\delta$-fields and normalize, see following, as to obtain the same symmetry energy at saturation.

The density dependence of the couplings, for all channels, for all the tested models is shown in Fig.1. In the $N L-R M F$ models the couplings are constant, for the $D D H-R M F$ model they are chosen as discussed above. Note, in particular, the unusual density dependence of the $\delta$-like vertex and the attempt to reproduce some average constant value in the corresponding $N L \rho \delta$ parametrization. Finally we like to remind that, due to the non-linear self interaction of the $\sigma$-meson, in the $N L-R M F$ models the effective $g_{\sigma}$ will also 
show a density dependence.

The symmetry energy $E_{\text {sym }}$ is defined from the expansion of the energy per nucleon $E\left(\rho_{B}, \alpha\right)$ in terms of the asymmetry parameter (we use $\rho_{B}$ for the baryon density in order to avoid confusion with the $\rho$ meson):

$$
E\left(\rho_{B}, \alpha\right)=E\left(\rho_{B}\right)+E_{\text {sym }}\left(\rho_{B}\right) \alpha^{2}+\mathcal{O}\left(\alpha^{4}\right)+\cdots
$$

with the abbreviation

$$
E_{\text {sym }}=\left.\frac{1}{2} \frac{\partial^{2} E\left(\rho_{B}, \alpha\right)}{\partial \alpha^{2}}\right|_{\alpha=0}=\left.\frac{1}{2} \rho_{B} \frac{\partial^{2} \epsilon}{\partial \rho_{3}^{2}}\right|_{\rho_{3}=0} .
$$

From the definition of the energy momentum tensor (6) one obtains for the symmetry energy $[15,16]$ :

$$
E_{\text {sym }}=\frac{1}{6} \frac{k_{F}^{2}}{E_{F}}+\frac{1}{2}\left[f_{\rho}-f_{\delta}\left(\frac{m^{*}}{E_{F}}\right)^{2}\right] \rho_{B} .
$$

where $E_{F}=\sqrt{k_{F}^{2}+m^{* 2}}$.

It is seen that including the scalar iso-vector $\delta$-meson the bulk asymmetry parameter is given by the combination $\left[f_{\rho}-f_{\delta}\left(\frac{m^{*}}{E_{F}}\right)^{2}\right]$ of the repulsive vector $(\rho)$ and attractive $(\delta)$ iso-vector couplings. We thus have to increase the $\rho$-meson coupling when including the $\delta$-meson in the iso-vector part of the equation of state in order to reproduce the same bulk asymmetry parameter $a_{4}$. This is a very general feature, not depending on the model, that we will see to be important in the dynamical simulations. Another $\delta$-effect, of interest for transport properties, will be the splitting of the neutron/proton effective masses $[15,16]$, see Eq. (7).

From Eq.(10) we note the moderate role of the scalar, iso-vector $\delta$-meson at low densities, and therefore on properties of finite nuclei (see below), as already pointed out in [23]. It is important to realize the different Lorentz structure of the iso-vector mesons. The vector $\rho$-meson contribution depends linearly on the baryon density whereas the scalar $\delta$-meson part is proportional to the scalar density or correspondly to the factor $\left(m^{*} / E_{F}\right)$, decreasing at high baryon density.

In Fig. 2 the density dependence of the symmetry energy, Eq. (10), for the different models used here is shown. The kinetic and the potential contributions to the total symmetry energy can be also seen separately on the bottom. Within the Walecka models $(N L \rho$ and $N L \rho \delta))$ one observes a similar behavior 

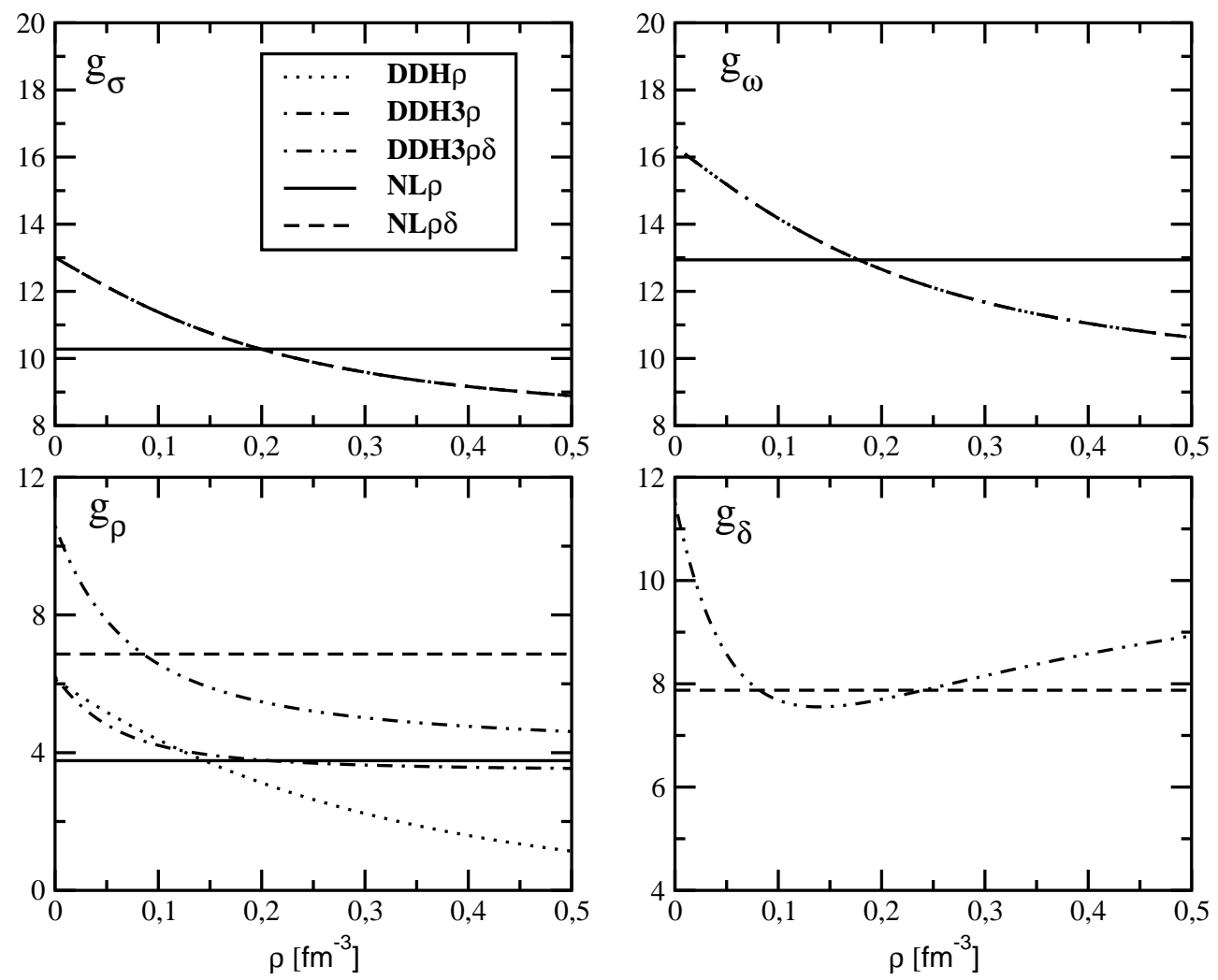

Fig. 1. Density dependence of the different coupling functions as indicated for the relativistic mean field models used here (see text for details).

of $E_{\text {sym }}$ at densities below saturation. With increasing baryon density $\rho_{B}$, however, the differences arising from the presence of the $\delta$-meson in the iso-vector channel become more pronounced, as expected. This is due to the saturation of the scalar density (the factor $\left(\frac{m^{*}}{E_{F}}\right)^{2}$ of Eq.(10)) for high densities and, on the other hand, to the enhanced value of the vector $\rho$-meson contribution (needed to reproduce the same $a_{4}$, see Table 1 ) which then grows linearly with baryon density.

In the $D D H$ models the high density influence of the $\delta$-meson on the isovector part of the equation of state is more involved. We have now a rapid decrease with density of the $\rho$-coupling function while the increase of the $\delta$-coupling above saturation almost counteracts the relativistic scalar density effect, see Fig.1. As we can see from the two DDH3 columns of Fig.2, with the inclusion of the $\delta$-meson the symmetry energy is not much changed for baryon densities higher than saturation and actually can become even softer. The Lorentz structure of the isovector contributions is now different, and moreover we have an important splitting of the $n / p$ effective masses. We will show that from particle production it may be possible to trace back these fine relativistic effects, although of course difficult. The density behavior of symmetry energy for the the $D D H \rho$ model, (last column of Fig.2), can be regarded as very soft originating from a relatively strong density decrease of the $\rho$-meson coupling, see Fig.1. This is particularly evident in the potential contribution (dashed line on the bottom-right). From Fig.2 the importance of relativistic features in the understanding of the high density behavior of the symmetry energy is 


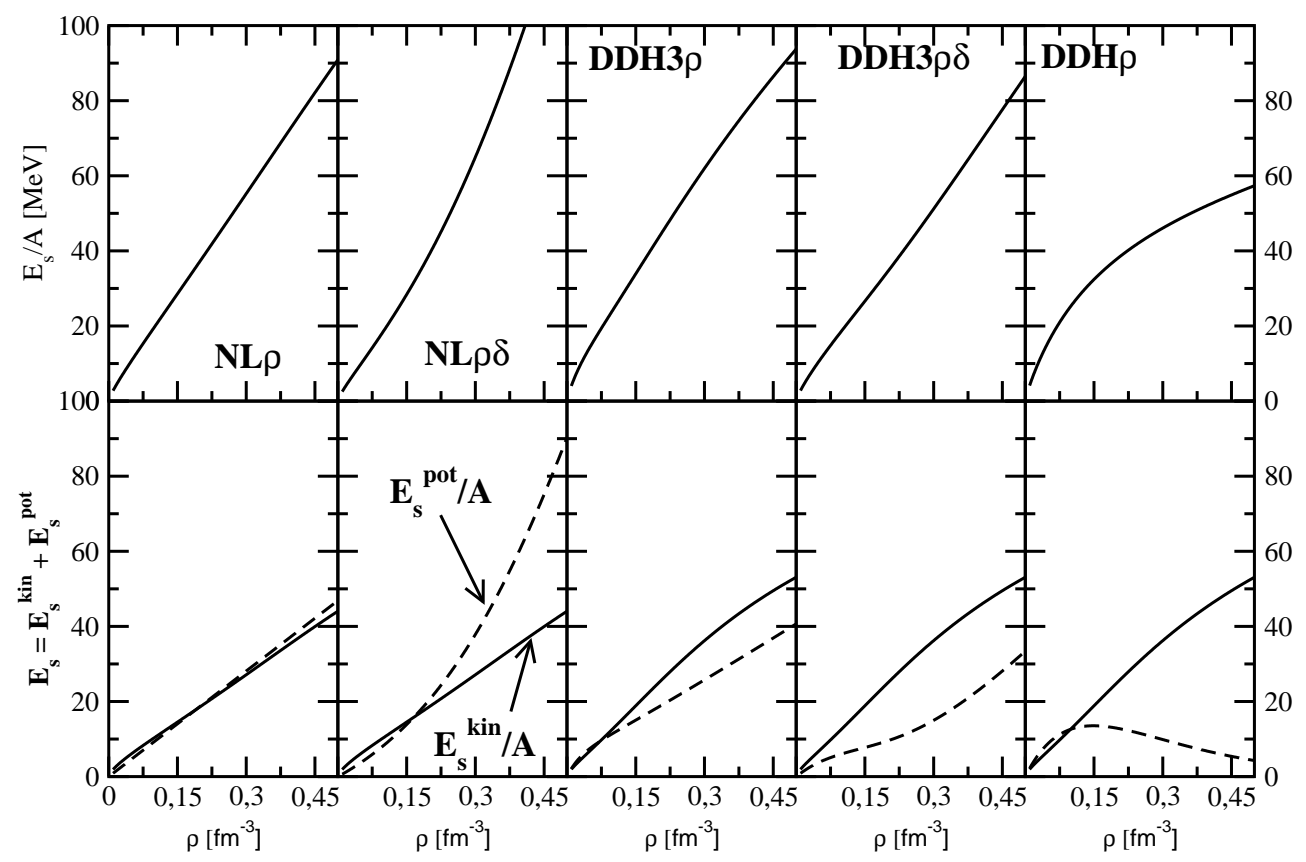

Fig. 2. Density dependence of the symmetry energy, Eq. (10), for the same models as in Fig. 1. The lower part shows separately the kinetic (solid) and potential (dashed) contributions to the total symmetry energy.

evident.

In contrast to the iso-scalar part of the mean field, which has been extensively investigated in the last three decades theoretically and experimentaly $[1,35]$, there is not enough empirical information about the high density behavior of the symmetry energy. Heavy ion collision experiments with radioactive beam facilities have recently been started or are being planned. In the following section we investigate first in finite nuclei the Lorentz structure of the isovector channel in the framework of the models discussed here. In the next section we will then pass to heavy ion collisions at intermediate energies, where higher densities can be reached and relativistic effects can be enhanced. We will discuss constant coupling Walecka-type models $(N L \rho$ and $N L \rho \delta)$ ) as a kind of reference to clearly see the physics of the relativistic effects of different isovector contributions. We will compare then with the more sophisticated $D D H \rho$ and $D D H 3 \rho, D D H 3 \rho \delta$ models in order make predictions for the main observables in experiments.

\section{$3 \quad$ Finite nuclei (static case)}

In this Section we focus on the role of the iso-vector, scalar $\delta$-meson in the properties of finite nuclei and, particularly, we compare with other models 

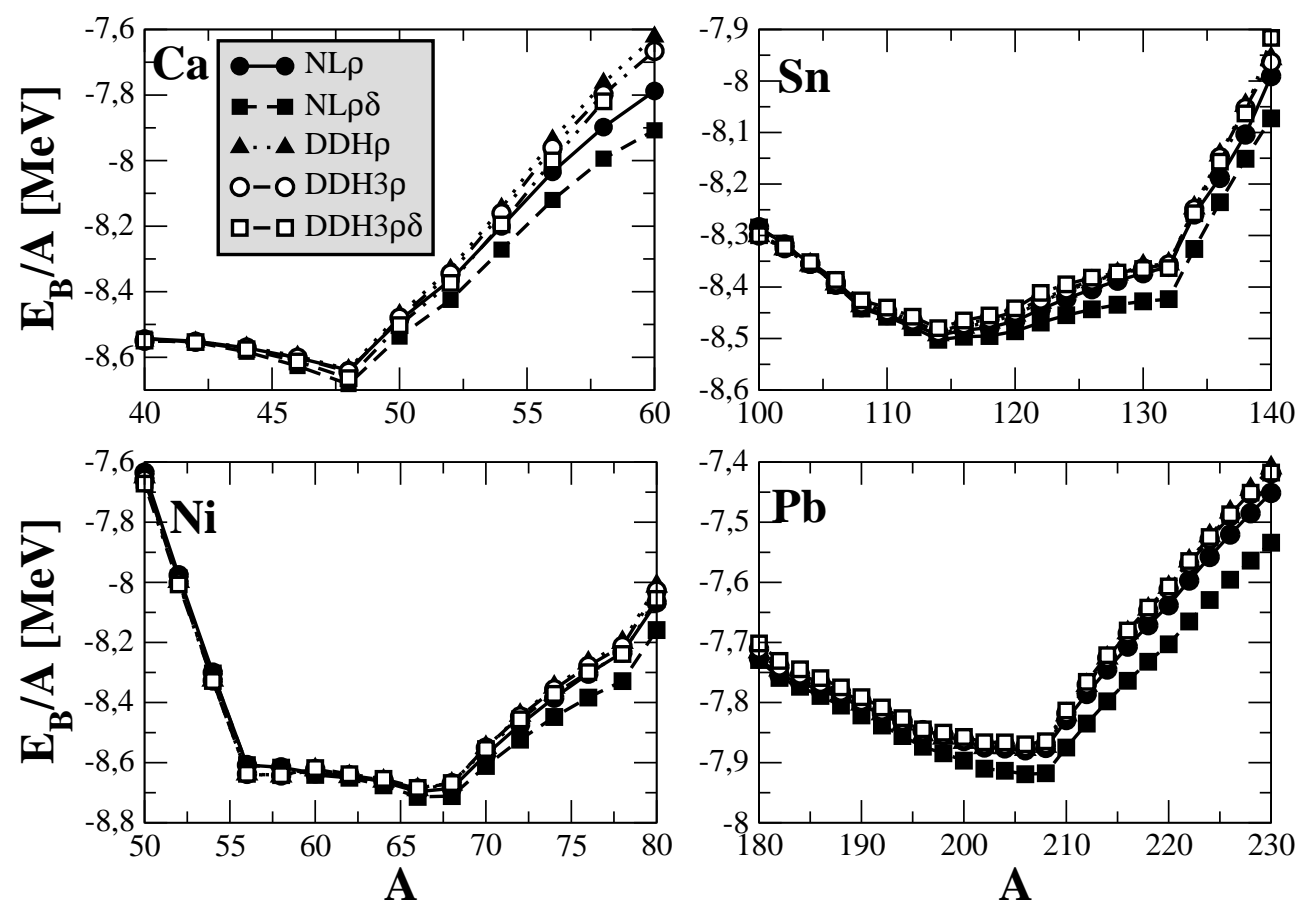

Fig. 3. Mass dependence of the binding energy per nucleon for $\mathrm{Ca}, \mathrm{Ni}, \mathrm{Sn}$ and $\mathrm{Pb}$ isotopes for the different models.

widely used in nuclear structure studies. For the description of finite nuclei we solve the equations of motion for nucleons and mesons, Eqs. (1-5), including the gradient terms in the classical meson field equations in a selfconsistent scheme. In case of density dependent coupling functions also additional rearrangement contributions of the iso-scalar and iso-vector classical fields (not shown in Eqs.(1-6)) are explicitely taken into account [23,19].

Fig. 3 displays the mass dependence of the binding energy per nucleon for different isotopes, starting from the most symmetric case $(\mathrm{Ca})$ to the most asymmetric one $(\mathrm{Pb})$. The results for all models are close together but the differences originating from the $\delta$-meson become more pronounced with increasing neutron to proton asymmetry. This is particularly evident for the constant coupling $R M F$ models, when comparing $N L \rho$ and $N L \rho \delta$ results.

We recall from Fig.2 that in the region around and below saturation, tested in finite nuclei, no significant differences between the models are seen in the symmetry energy. Only between the two extreme cases of $N L \rho \delta$ and $D D H \rho$ there is a visible difference in the low density regime which also leads to effects in the binding energy per nucleon for highly asymmetric finite systems. Generally, the binding energy becomes larger with the $\delta$-meson included in the iso-vector mean field because here the attractive mean field is stronger (smaller) for neutrons (protons), with an observed net effect for neutron-rich nuclei. The comparison with the widely used models $D D H \rho$ [23] and $N L 3$ $[31,32]$ (here essentially given by the $N L \rho$ points), which nicely describe finite 

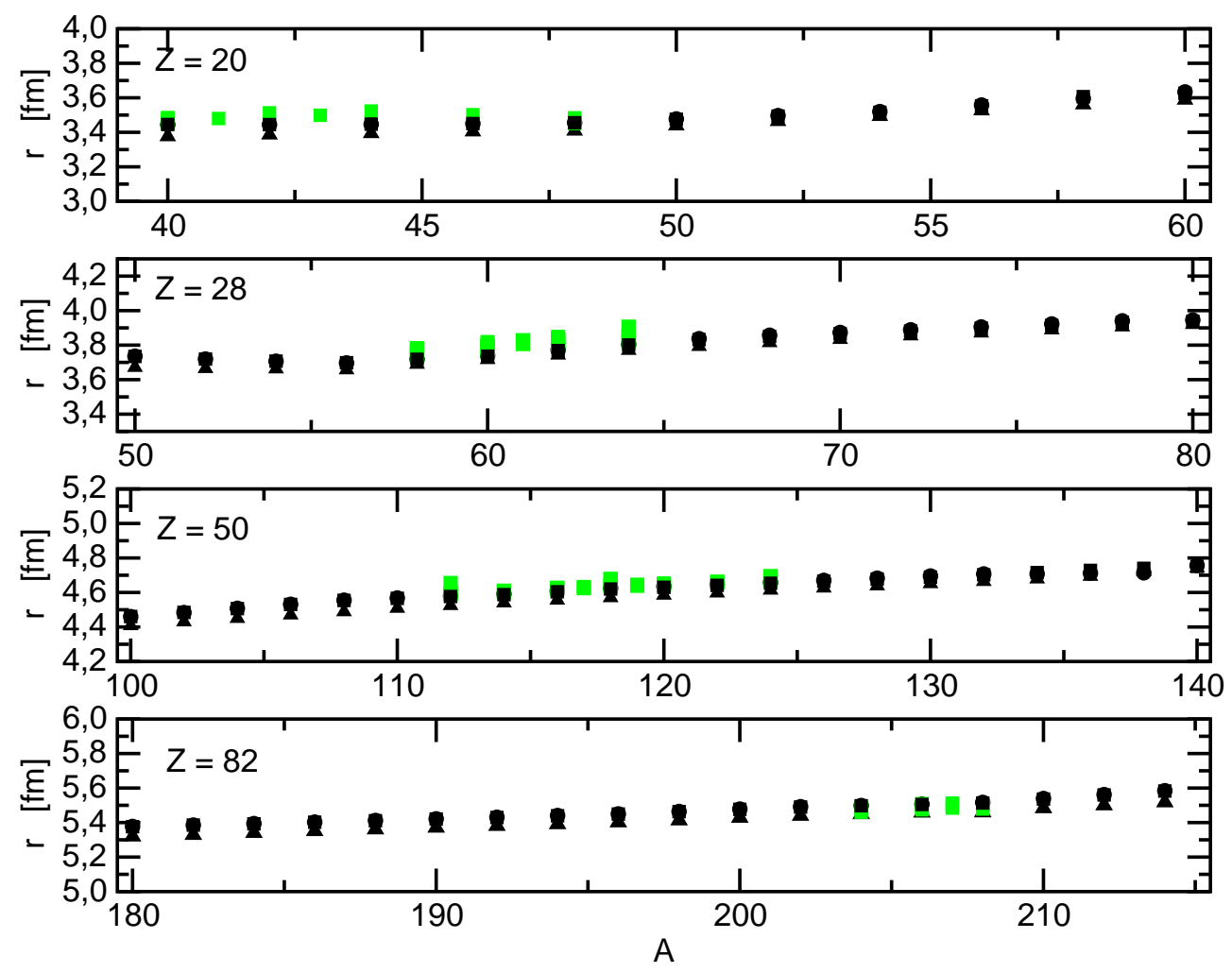

Fig. 4. Mass dependence of charge proton radii for $\mathrm{Ca}-, \mathrm{Ni}-, \mathrm{Sn}-$ and $\mathrm{Pb}$-isotopes: (grey squares) exp. data, (full triangles) $D D H \rho$, (open triangles) $N L 3$, (full circles) $N L \rho$ ) and (full squares) $N L \rho \delta)$.

nuclei properties, indicates the consistency of our parametrizations, that we will then extend to the description of heavy ion collisions.

Fig. 4 shows the charge proton radii for different isotopes, as indicated. First, one observes moderate differences between the different models, since the proton density profiles are very similar, as expected. All the models can reproduce the experimental values, as an important issue.

One should expect larger effects of the internal structure of the symmetry energy by looking at the difference between neutron and proton radii, which is more sensitive to slope and curvature of the symmetry energy around saturation $[11,12]$. This is shown in Fig. 5 for the same models and systematics. As in the previous figures, the influence of the $\delta$-meson is again not much pronounced in the $N L-$ and $D D H$-models, because of the similar density behavior of the symmetry energy at low densities. We can see a systematic smaller $r_{n}-r_{p}$ for neutron-rich nuclei, i.e. a reduced neutron diffusivity, in the case of $D D H$ parametrizations, due to the larger $\rho$-(symmetry energy) repulsion seen by neutrons on the surface (at lower densities), see Fig.1. This seems to be in better agreement with data, see [12]. We note that this result is present also when the $\delta$ channel is added, see the open squares of the $D D H 3 \rho \delta$ interaction, in particular for the largely discussed ${ }^{208} \mathrm{~Pb}$ case.

At variance the slightly smaller symmetry repulsion for neutrons at densities 

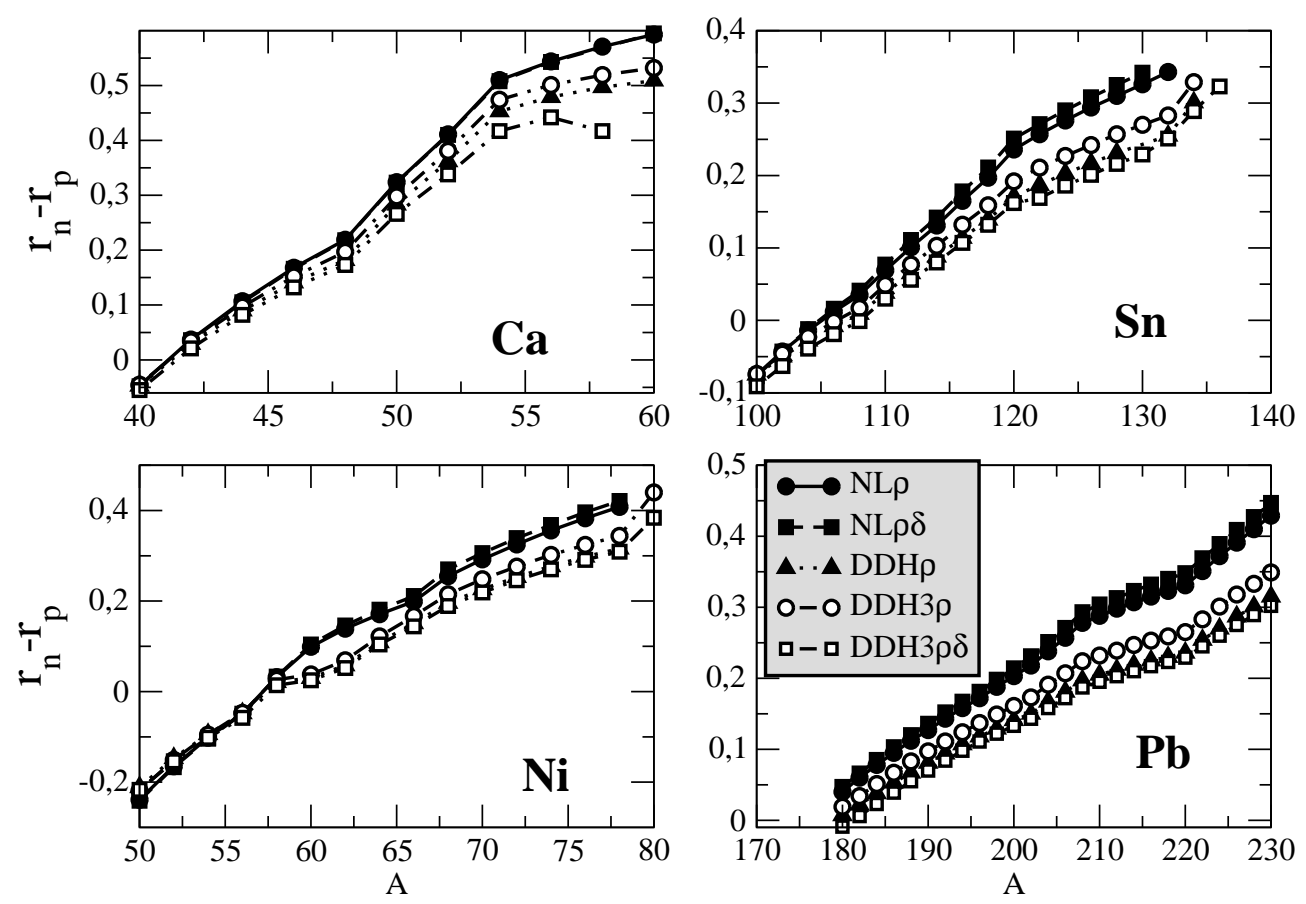

Fig. 5. Mass dependence of the difference of the neutron to proton radii for the same isotopes and models as in Fig. 3.

below $\rho_{0}$ in the $N L \rho \delta$ parametrization can consistently account for the larger neutron diffusion and the overall larger binding energies for very neutron-rich nuclei, Fig.3.

This discussion confirms the relatively small effect of a more extended relativistic structure of the symmetry term in the low density range, tested in finite nuclei structure calculations, see also [23]. Similar results have been observed in the spin-orbit splitting of single particles levels, which are not shown here.

Generally the properties of finite nuclei can be well reproduced in the $N L$ models and are close to the results of more realistic $D D H$ approaches [23]. This is an important test of the $R M F$ parametrizations before applying them to the dynamics of heavy ion collisions. However, it seems difficult to determine the relativistic structure of the iso-vector part of the nuclear mean field in the static case, because the influence of the $\delta$-meson is only moderate, except if one goes to extremely asymmetric neutron-rich nuclei. However, this is not the aim of the paper, but the behavior of the symmetry energy at supra-normal densities. Since in heavy ion collisions highly compressed matter is formed, at least for short time scales, we study now the dynamical case in more detail. The high velocity fields will also enhance the different Lorentz properties of the various contributions, [25]. 


\section{Heavy ion collisions (dynamical case)}

The theoretical treatment of heavy ion collisions at relativistic energies between 0.1 and $1-2 A G e V$ is based here on a covariant transport theory. As detailed described in Ref. [36], starting from a covariant quantum description of the nuclear many body problem one arrives at a transport equation which is called in the literature Relativistic-Boltzmann-Uhlenbeck-Uehling ( $R B U U)$ equation

$$
\begin{aligned}
& {\left[k^{* \mu} \partial_{\mu}^{x}+\left(k_{\nu}^{*} F^{\mu \nu}+m^{*} \partial_{x}^{\mu} m^{*}\right) \partial_{\mu}^{k^{*}}\right] f\left(x, k^{*}\right)=} \\
& =\frac{1}{2} \int \frac{d^{3} k_{2}}{E_{k_{2}}^{*}(2 \pi)^{3}} \frac{d^{3} k_{3}}{E_{k_{3}}^{*}(2 \pi)^{3}} \frac{d^{3} k_{4}}{E_{k_{4}}^{*}(2 \pi)^{3}} W\left(k k_{2} \mid k_{3} k_{4}\right)(2 \pi)^{4} \delta^{4}\left(k+k_{2}-k_{3}-k_{4}\right) \\
& \times\left[f\left(x, \mathbf{k}_{3}\right) f\left(x, \mathbf{k}_{4}\right)(1-f(x, \mathbf{k}))\left(1-f\left(x, \mathbf{k}_{2}\right)\right)-\right. \\
& \left.\quad f(x, \mathbf{k}) f\left(x, \mathbf{k}_{2}\right)\left(1-f\left(x, \mathbf{k}_{3}\right)\right)\left(1-f\left(x, \mathbf{k}_{4}\right)\right)\right],
\end{aligned}
$$

with effective masses $m^{*}$, effective momenta $k^{* \mu}$ and the field tensor defined by $F^{\mu \nu}=\partial_{x}^{\nu} \Sigma^{\mu}-\partial_{x}^{\mu} \Sigma^{\nu} . \Sigma^{\mu}$ are the vector self-energies and $W\left(k k_{2} \mid k_{3} k_{4}\right)$ the transition rate for the process $k+k_{2} \rightarrow k_{3}+k_{4}$.

The main approximations involved in Eq. (11) are a semi-classical gradient expansion and a quasi-particle approximation which sets the effective momenta on shell, i.e. $k^{* \mu} k_{\mu}^{*}=m^{* 2}$. The numerical procedure is performed within the relativistic Landau Vlasov method $[37,38]$, where the phase space distribution function $f\left(x, k^{*}\right)$ is characterized by test particles of a covariant Gaussian shape in coordinate and momentum space. Sufficient stability is achieved when using $\sim 40-50$ test particles per nucleon for the simulations.

Protons and neutrons are propagating separately according to their hadronic fields and the Coulomb interaction. The collision integral is treated by MonteCarlo methods [39] including all important inelastic channels up to $1-2 \mathrm{AGeV}$ [40]. Here we explicitely propagate $\Delta^{0, \pm,++}-$ and $N^{*}$-resonances including their decay to one- and two-pion $\left(\pi^{0, \pm}\right)$ channels. At these energies we consider the lower mass resonances only, with free mass assignements $M_{\Delta}=1232 \mathrm{MeV}$ and $M_{N^{*}}=1440 \mathrm{MeV}$. For elastic scattering we apply the empirical evaluations of isospin dependent cross sections [41]. For the inelastic channels we use the free cross section parametrizations of ref. [42]. The produced pions are also propagated under the influence of the Coulomb field. We should mention that the parameters in the collision term are commonly used by other groups. Main properties as rapidity distributions and transverse momentum spectra of protons as well as pion multiplicities are in agreement with results from

other groups when using similar parameters for the nuclear equation of state 

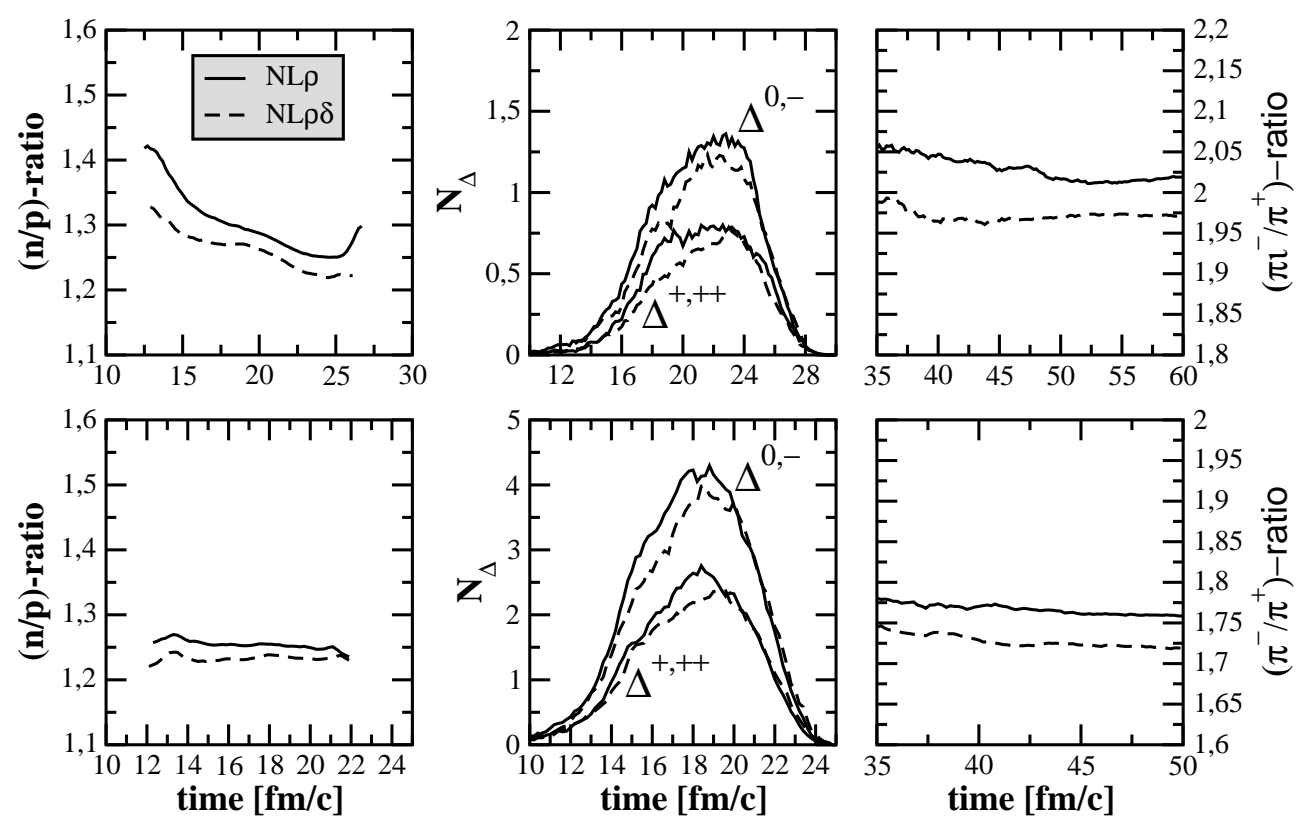

Fig. 6. Time dependence of the $\left(\frac{n}{p}\right)$-ratio (left), the multiplicities of $\Delta^{0,-}, \Delta^{+,++}$ resonances (middle) and the $\left(\pi^{-} / \pi^{+}\right)$-ratio (right) for central $(b<2 \mathrm{fm}) A u+A u$ collisions at 0.6 (top) and $1 A G e V$ (bottom). Results of $R B U U$ calculations with the $\rho$-meson ( $N L \rho$, solid) only and both, the $\rho$ and $\delta$ mesons ( $N L \rho \delta$, dashed) for the isovector channel are shown.

$[43,44]$.

It has been suggested since some time that the difference in neutron-proton flows should be a sensitive observable to study the density behavior of the isovector EOS. Several calculations in the non-relativistic framework have indeed shown such sensitivity to the asymmetry-stiffness, but rather extreme choices were involved, see refs. [14,45]. In a previous letter [25] we have investigated proton-neutron differential flows in the framework of the present $R M F$ models. Also in this case a strong sensitivity was found, at high energies, clearly due to the Lorentz structure of the isovector interaction. However no data exist at present. In this work we will concentrate on pion production and spectra. We are also motivated by the fact that recent experimental results on isospin effects in pion production are starting to appear, $[46,47]$.

\subsection{Neutron/proton densities and $\pi^{-} / \pi^{+}$ratios}

Observable effects originating from the high density symmetry energy should be related to differences on ratios of neutron and proton densities. Thus, we consider in the following the ratio of neutrons to protons as a function of time and space. One also expects isospin effects on different isospin channels of 
pions, since they are produced via $n n, p p$ and $n p$ collisions through the decay of $\Delta$ and $N^{*}$ resonances.

Fig. 6 shows the time evolution of the ratio of neutrons to protons for regions of density higher than saturation, of the multiplicities of $\Delta^{0,-}$ and $\Delta^{+,++}$resonances and finally of the ratio of negative to positive pions for central (impact parameter $b<2 \mathrm{fm}$ ) $A u+A u$ collisions at 0.6 and $1.0 A G e V$ using the $N L$ models without (solid) and with (dashed) the $\delta$ field. In the $N L \rho \delta$ calculations the neutrons are emitted much earlier than protons from the high density phase due to a more repulsive mean field joined to a lower $n$-effective mass. This effect is responsible for the reduction of the $n / p$-ratio in the residual system and, particularly, it influences the particle production, see also [24,48].

The four isospin states of the $\Delta$-resonance are produced from different scattering channels, when the threshold energy is available, e.g. $n n \rightarrow p \Delta^{-}, n \Delta^{0}, p p \rightarrow$ $p \Delta^{+}, n \Delta^{++}, \cdots$. Thus $\Delta^{0,-}\left(\Delta^{+,++}\right)$resonances are mainly formed in energetic $n n-(p p-)$ collisions. Therefore, the sensitivity on the density dependence of the symmetry energy of the $n / p$-ratio is indirectly related to that of the particle production.

The $n / p$ effective mass splitting in asymmetric matter can also directly affect the resonance production around the threshold, with noticeable effects on the yields. We remind that resonances will also have isospin dependent in-medium effective masses that can be roughly related to the nucleon effective masses just using the isopin coupling coefficients in the process $\Delta \leftrightarrow \pi N$ [24]. Thus the effective masses of the four isospin states of the $\Delta$-resonance will be different when the $\delta$-meson is included in the calculation. In any case we know from general grounds, see Section 2, that, in $n$-rich systems, a isovector scalar meson field is leading to a neutron effective mass smaller than proton, in particular at high baryon densities. Consequently in the process $n n \rightarrow p \Delta^{-}$less energy will be available for the $\Delta^{-}$production in the $N L \rho \delta$ case.

We like to note that this mechanism is not necessarily linked to the density behavior of the symmetry energy. E.g., in density dependent coupling models we can have at high densities a soft $E_{\text {sym }}(\rho)$ just because the $\rho$-meson coupling is decreasing but still a large $m_{p}^{*}-m_{n}^{*}$ splitting if the $\delta$-meson coupling stays constant or slightly increasing, like in the $D B H F$ estimations (see Fig.1).

In the resonance multiplicity (middle figures) one thus sees a decrease of the $\Delta^{0,-}$ isospin states due to the effect of the isovector-scalar $\delta$-meson. The pions, on the other hand, are mainly produced from resonance decay according, e.g. $\Delta^{-} \rightarrow n \pi^{-}, \Delta^{0} \rightarrow p \pi^{-}, \Delta^{+} \rightarrow n \pi^{+}, \cdots$ Therefore the decrease of $\Delta^{0,-}$ resonances reduces the production of negative charged pions which consequently decreases the $\pi^{-} / \pi^{+}$-ratio (right part in Fig. 6 ). This ratio then appears to be sensitive to the isospin term of equation of state at high densities. However it 

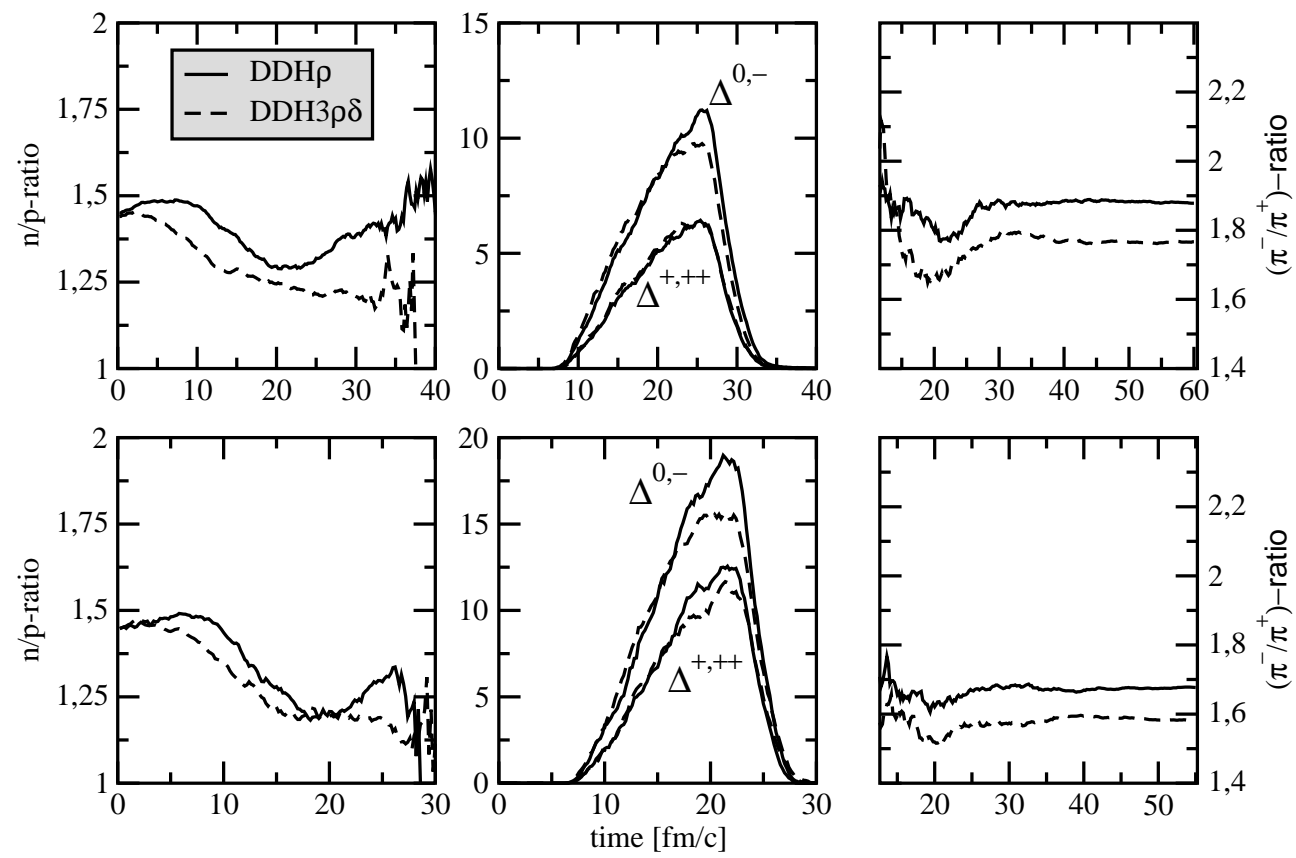

Fig. 7. Same as in Fig. 6, but with the $D D H \rho$ (solid) and $D D H 3 \rho \delta$ (dashed) models where the isovector couplings are explicitely density dependent. Note the larger $y$-scales compared to the $N L$ cases of Fig. 6 .

is also affected by secondary pion absorption channels which start to influence the results at high energies.

One should note that both $N L$ models used here exhibit a similar asy-stiff behavior at high densities (see Fig.2). We can deduce that the effective $n / p$ mass splitting mechanism described before is very important in inducing isospin effects on the particle production [49].

Next Fig. 7 shows the same quantities as in Fig.6 but now using the $D D H$ models where all coupling functions are density dependent. In this case we have compared the $D D H \rho$, very soft symmetry energy, with $D D H 3 \rho \delta$, relatively stiff one. Due to the strong density decrease of the $\rho$ - coupling function in the $D D H \rho$ parametrization, now the influence of the introduction of the isovector $\delta$ channel is more pronounced comparing to the $N L$ cases, because of the larger differences in the high density symmetry energy and in the $n / p$ effective mass splitting. In the transport calculations with the $\delta$ meson (leftcenter panels of Fig.7) the $n / p$-ratio and the $\Delta^{-}$-yield decrease at high density is indeed stronger than in the $N L$ models (Fig.6). Consequently we also see a stronger influence on pion production.

By comparing the Figs. 6 and 7 we see clear differences in the total multiplicity of resonances and therefore in the absolute pion yields. One should note that the $D D H$ parametrizations differ from the $N L$ models also in the iso-scalar 
part of the mean field. In particular, the EOS for symmetric nuclear matter, which is similar for both models around saturation, has a smaller incompressibility at $\rho_{0}$ in the $D D H$ cases (see the discussion in Sect.2) and it is also softer at high densities $[19,23]$. Thus, in the dynamic calculations with the $D D H$ parametrizations one naturally obtains more compression in the central region of the reaction which, in turn, is responsible for the production of higher multiplicities of resonances. The factor $5-7$ difference in the $\Delta$ formation probability during the compression phase, see the middle panels in Figs.6- 7, seem to indicate that pion absolute yields can be a good probe of the nuclear EOS at high densities, apart from corrections due to in-medium modifications of inelastic cross sections, resonance decays and $\pi$-reabsorption effects, see ref.[43].

The comparison between the $D D H$ and $N L$ models with respect to isospin effects depends on several factors. However, the reason of the presentation of these different models was to focus on the effects originating from the introduction of the scalar iso-vector $\delta$ field in the symmetry energy. We have shown that there are common features in all models, namely a larger repulsion and a smaller effective mass for neutrons in high baryon and isospin density regions, for $n$-rich systems. A decrease of the $n / p$ ratios and of the $\Delta^{-, 0}$ multiplicities will finally reduce the charged pion $\pi^{-} / \pi^{+}$ratios.

\subsection{Isospin diffusion}

Before we continue to discuss the pion yields in more detail, we briefly make some further comments on the $n / p$ ratios in different density regions. This is related to the isospin diffusion from high to low density regions and with the subsequent formation of fragments. This has been suggested in various works $[13,27]$ as another means of investigating the density dependence of the isovector EOS. In Fig.8 we show the time evolution of the $n / p$ ratio in regions with density below (upper panels, "gas and light clusters") and above (lower panels, "liquid") saturation density for two incident energies. In the upper panels we also show the total central baryon density as an indicator of the global evolution of the collision. We use the extreme density dependent coupling models as in Fig.7, namely the very asy-soft $D D H \rho$ (solid) and the asy-stiff $D D H 3 \rho \delta$ (dashed) interactions.

One sees in the upper panels that there is a strong emission of neutrons to the gas just before and at maximum compression for the asy-stiff model, where there is a larger differential driving force on the neutrons. The effect is essentially non existing for the asy-soft model. In correspondence the $n / p$ ratio of the liquid (lower panels) decreases below the initial value, more so for lower energies. Consequently the chemical composition of the light clusters rapidly 

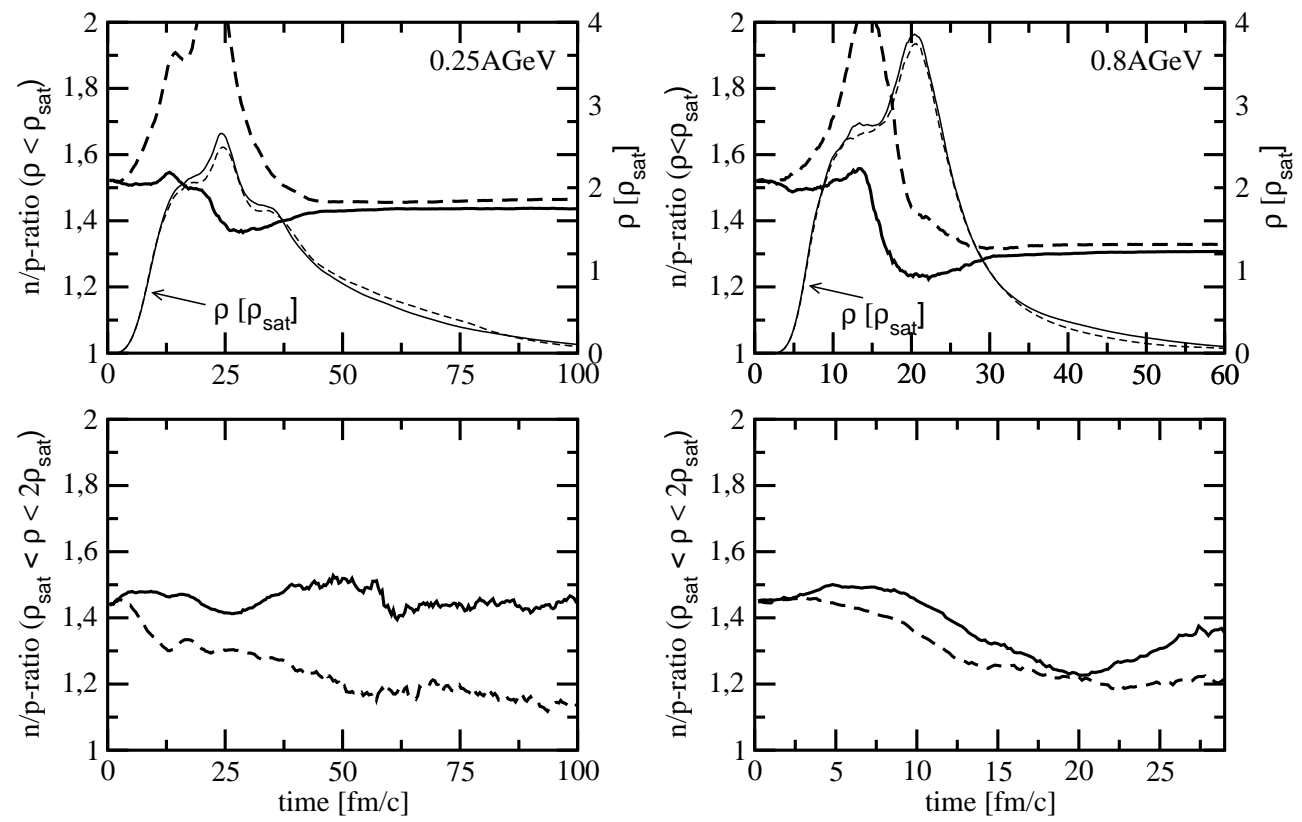

Fig. 8. Time evolution of the $n / p$-ratio (left scale) for density regions below (upper panels) and above (lower panels) saturation density in $A u+A u$ collisions at energies of 0.25 (left panels) and $0.8 \mathrm{AGeV}$ (right panels). The upper panels also show the evolution of the central total densities in units of $\rho_{\text {sat }}$ (thin lines, right hand scale). The models shown are the same as in Fig.7: $D D H-\rho$ (solid lines) and $D D H 3 \rho \delta$ (dashed).

formed during the expansion phase will show signatures of the asy-stiffness of the EOS, as already suggested in ref.[27]. However, at later times the $n / p$ ratio of the dilute region is very similar for the two interactions (slightly below the initial value), meaning that at these energies the bulk of the matter finally ends up in free nucleons and light clusters.

\subsection{Energy dependence of $\pi^{-} / \pi^{+}$-ratios}

We now take up a more detailed discussion of isospin effects on pion yields. It was see in Figs.6,7 that the $\pi^{-} / \pi^{+}$-ratios stabilize after the compression phase and that they depend on the isovector EOS and, particularly, on the inclusion of the $\delta$-field. The latter is generally leading to a more asy - stiff EOS and in any case to a reduction of the neutron effective mass vs. the proton one. The larger neutron repulsion produces less neutron rich matter, which in turn produces less negative charged $\Delta$-resonances. The same effect is directly reached by the reduction of the neutron effective mass. Correspondly less negative pions are formed, leading to a reduction of the $\pi^{-} / \pi^{+}$-ratio of the order of 5 to $10 \%$. 


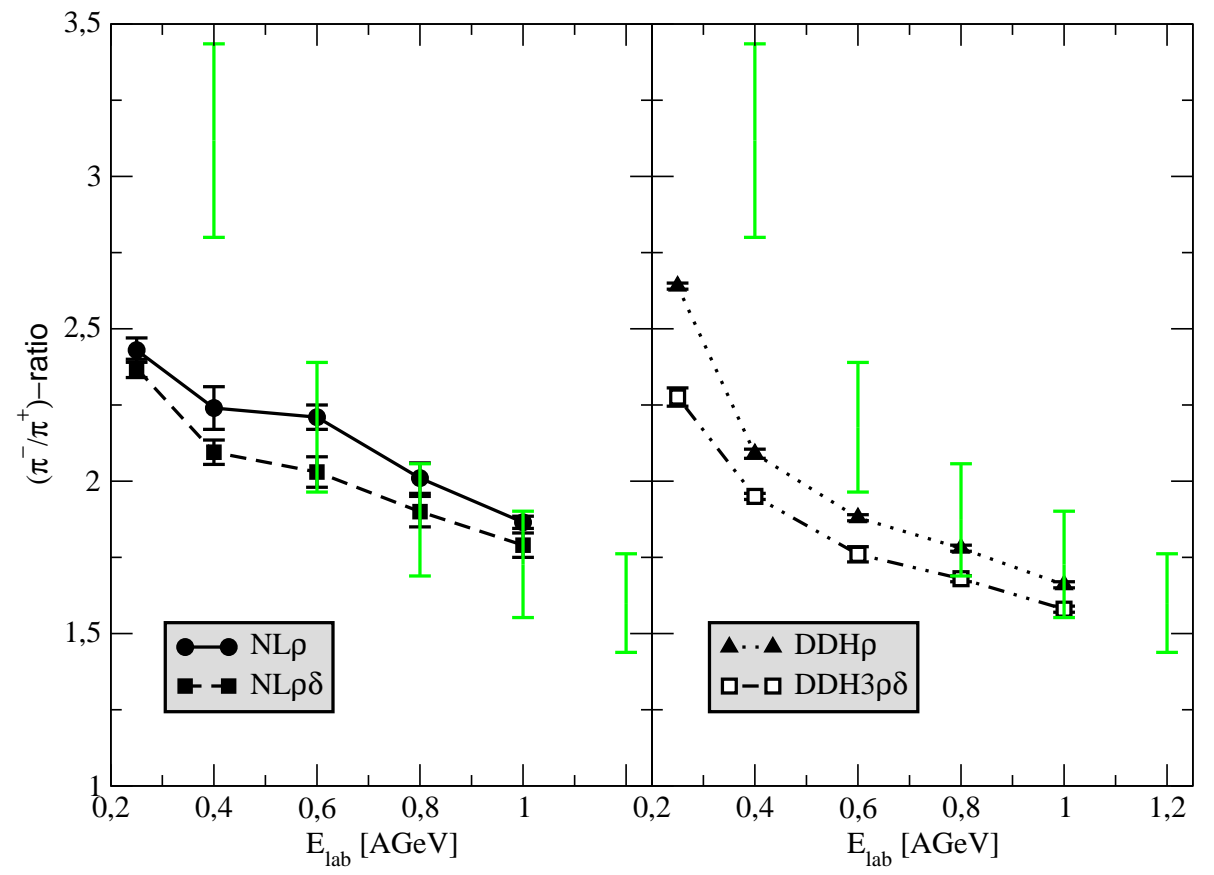

Fig. 9. Energy dependence of the $\left(\pi^{-} / \pi^{+}\right)$-ratio in central $A u+A u$ collisions. Left panel: $N L$ results, $N L \rho$ (solid) and $N L \rho \delta$ (dashed). Right panel: $D D H$ results, $D D H \rho$ (solid) and $D D H 3 \rho \delta$ (dashed). The grey bars correspond to some very preliminary data from the FOPI - GSI collaboration [46].

All the isospin effects on pion production are generally decreasing with incident energy, see Fig.9, due to secondary collisions, i.e. pion absorption and $\Delta$ rescattering. Thus all the isospin dependence of pion production will be on average moderated. Such effect was already found in earlier studies using a non-relativistic Quantum-Molecular Dynamics ( $Q M D)$ approach [48].

The beam energy behavior of the $\pi^{-} / \pi^{+}$-ratio evaluated at the freeze - out time for $A u+A u$ central reactions are shown in Fig.9, for the $N L$ models (left panel) and for the $D D H$ models (right panel). We like to note that in both cases the $\delta$ influence, difference between solid and dashed curves, is decreasing with the beam energy. This is a nice indication of the $n / p$ effective mass splitting mechanism in $\Delta^{-}$production, expected to be more important at the threshold.

For the same systems new very preliminary data on pion production have recently been reported from the FOPI-collaboration at GSI [46], shown as grey bars in the Fig.9. These experimental indications are used to test our miscroscopic evaluations.

We see that in our simulations we generally have a qualitative good description 


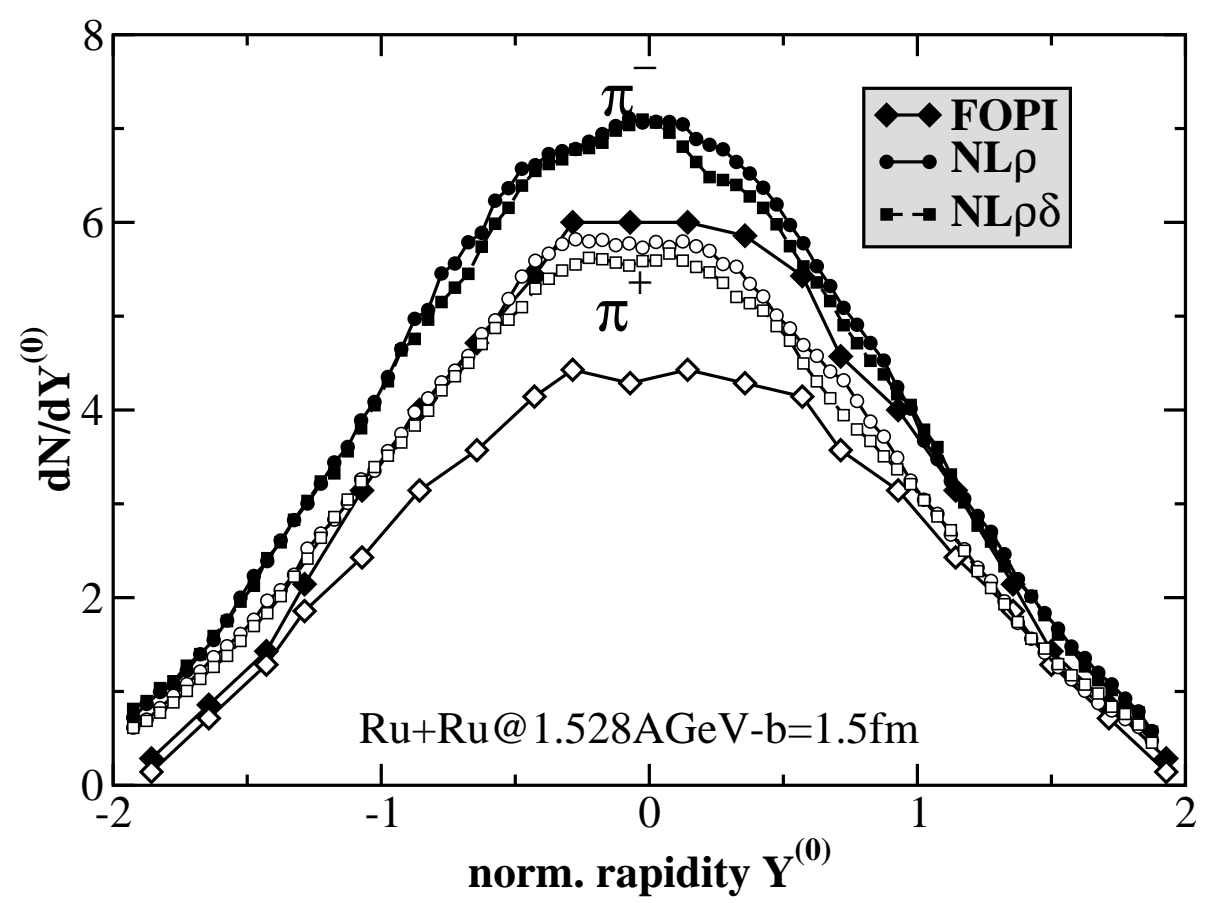

Fig. 10. $\pi^{ \pm}$-rapidity distributions for central ${ }^{96} R u+{ }^{96} R u$ collisions with $N L \rho$ and $N L \rho \delta$ models, without the Coulomb field for pion propagation. Full symbols are for $\pi^{-}$, open ones for $\pi^{+}$. Data are taken from Ref. [50].

of the pion ratio with respect to beam energy, except perhaps at the lower energies. The inclusion of a $\delta$ meson in the iso-vector part of the equation of state seems to improve the comparison, at least at higher energies. In any case it appears that the $\pi^{-} / \pi^{+}$-ratios might be a probe to the role of the high density symmetry energy and the role of the virtual $\delta$-meson. It would be nice to look at more exclusive data on pion production in asymmetric systems. This will be the subject of the next section on pion flows.

\section{$5 \quad$ Isospin effects on pion flows}

We now discuss in more detail the velocity distributions of pions, i.e. flow results. In spite of the relatively low asymmetry of the system $(N / Z=1.18)$ we will study central ${ }^{96} R u+{ }^{96} R u$ collisions at about $1.5 \mathrm{AGeV}$, since recent data are available from the FOPI collaboration [50]. We will show symmetry energy effects and how in this respect the Coulomb interaction in pion propagation is important. In particular we can expect the role of the Coulomb field to be not negligible in high density regions, for the interaction between the produced pions and the charged hadronic enviroment. We will also see that without taking the Coulomb effects into account, the differences originating from the $\delta$ meson are largely reduced. 


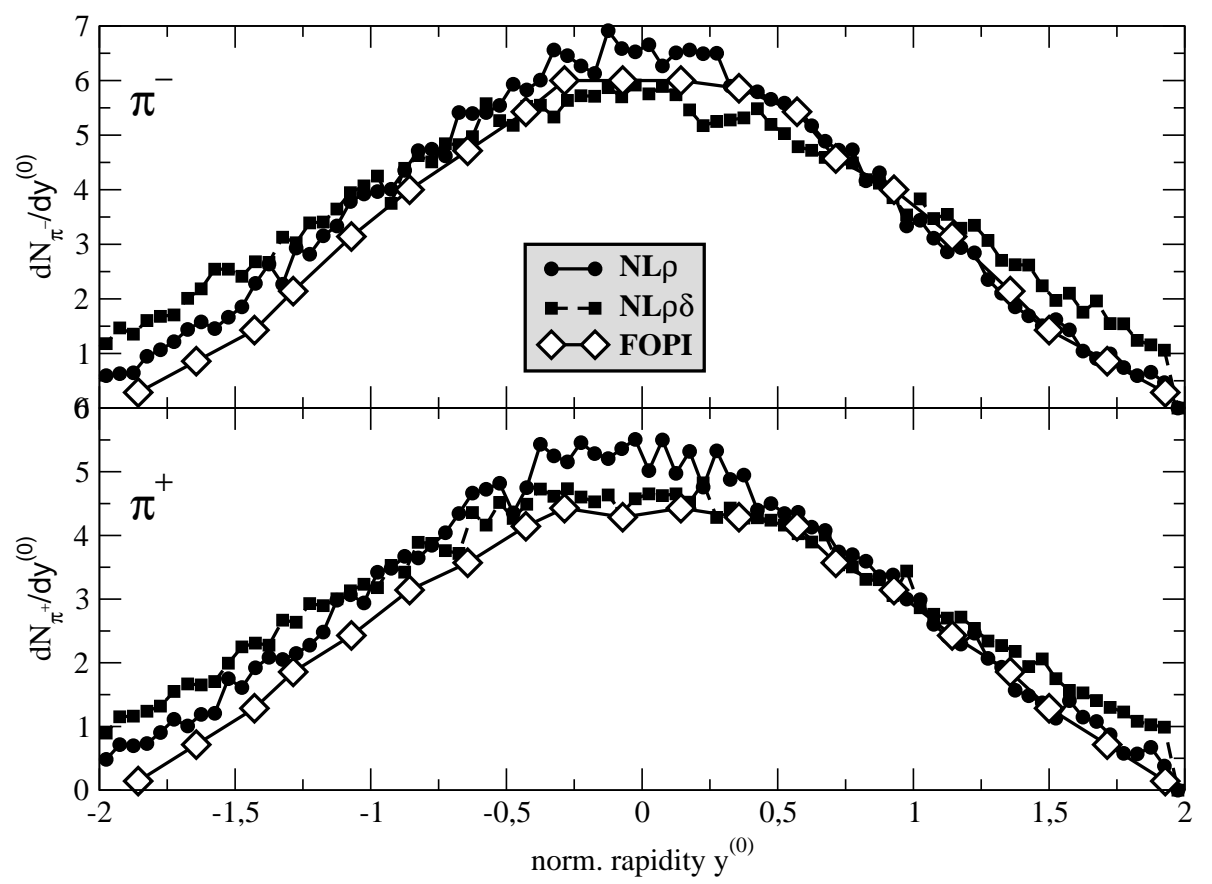

Fig. 11. $\pi^{ \pm}$-rapidity distributions for the same system as in Fig. 10, for the $N L \rho$ (full circles) and $N L \rho \delta$ (full squares) models, but including the Coulomb field in the pion propagation. Upper panel: $\pi^{-}$. Lower panel: $\pi^{+}$. Data (open diamonds) are taken from Ref. [50].

In Figs.10 and 11 we show the rapidity distributions for $\pi^{ \pm}$in comparison to the data for the $N L \rho$ and $N L \rho \delta$ models. The normalized rapidity is defined by $Y^{0} \equiv\left(Y / Y_{\text {proj }}\right)_{c m}$.

In the calculations of Fig.10 the Coulomb interaction was turned off for the pion propagation. It is seen that without the Coulomb interaction the rapidity distribution is generally too high compared to experiment, particularly so at mid-rapidity. Including the Coulomb, Fig.11, the distributions are reduced, in much better agreement with the data. The effect of including $\delta$-fields is also enhanced.

During the high compression stage the Coulomb field accelerates the positively charged pions away from mid-rapidity. The opposite is happening with the $\pi^{-}$'s that will be attracted towards the higher density region where they will suffer a larger absorption. This leads to a broadening of the pion distributions and to a general reduction due to the enhanced rescattering and absorption. Similar effects were also seen in the non-relativistic calculations of ref.[48].

The combined role of the $\delta$ - and Coulomb- fields on the difference between positively and negatively charged pions is a more subtle effect. As we have seen previously the structure of the symmetry term affects the isospin diffusion from 
high to low density regions. Moreover the $\delta$ contribution is directly reducing the negative $\Delta$-resonance production through the $n / p$-mass splitting. As a consequence the $N L \rho \delta$ model leads to a more positively charged hadronic central region and to larger Coulomb effects, The freeze-out $\pi^{-} / \pi^{+}$ratio at mid rapidity is then reduced. However, the Coulomb field, acting more repulsive, is inducing larger reabsorption effects for high momentum $\pi^{+}$. As a consequence we can have an enhancement of the $\pi^{-} / \pi^{+}$ratio for high-rapidity pions. The influence of the $\delta$-channel is thus observable in terms of pion stopping.

This can be seen in Fig.12 (left panel), where we show the rapidity dependence of the $\pi^{-} / \pi^{+}$-ratio for the two $N L$ models. For mid-rapidity the inclusion of the $\delta$-field reduces the ratio with the mechanisms described before. Since the mid-rapidity pions are the most abundant (Fig.11), this is consistent with the global reduction of the $\pi^{-} / \pi^{+}$-ratio (see Fig.9, left panel).

\subsection{Transverse momentum dependence}

All the effects discussed before should be particularly important in the compressional phase of the collision, while pions are produced all over the reaction evolution up to the freeze-out. A good selection procedure will be to look at particles with high transverse momenta $P_{t}$. It is indeed physically intuitive, and confirmed by simulations $[25,48,51]$, that particles emitted from the earlier high density phase have higher $P_{t}$ 's.

The $P_{t}$-dependence of the $\pi^{-} / \pi^{+}$-ratio in central $R u+R u$ collisions for midrapidity pions is shown in the right panel of Fig.12. It is seen that the inclusion of the $\delta$-field strongly enhances the ratio for high- $P_{t}$ pions, bringing it into more agreement with the data.

It is here that one sees the combined symmetry and Coulomb effects most clearly. With inclusion of the $\delta$-field the high density phase is relatively more rich in protons and positively charged resonances. The Coulomb force accelerates $\pi^{+}$away from this region and so reduces their number at mid-rapidity. All that then enhances the $\pi^{-} / \pi^{+}$-ratio for high- $P_{t}$ particles.

We have repeated the analysis for the two $D D H$ interactions already studied before, $D D H \rho$ and $D D H 3 \rho \delta$. The inclusion of the $\delta$-channel leads to the same effect. We see an increase of the $\pi^{-} / \pi^{+}$-ratio for high- $P_{t}$ pions at mid-rapidity, as observed in experiments.

We have shown that $\pi^{-} / \pi^{+}$-ratios for high transverse momentum pions are a sensitive probe to the structure of the symmetry term, in particular to the contribution of a $\delta$-field. The trend of the data with rapidity and transverse momentum is affected by the dynamical effects of the inclusion of a isovector 

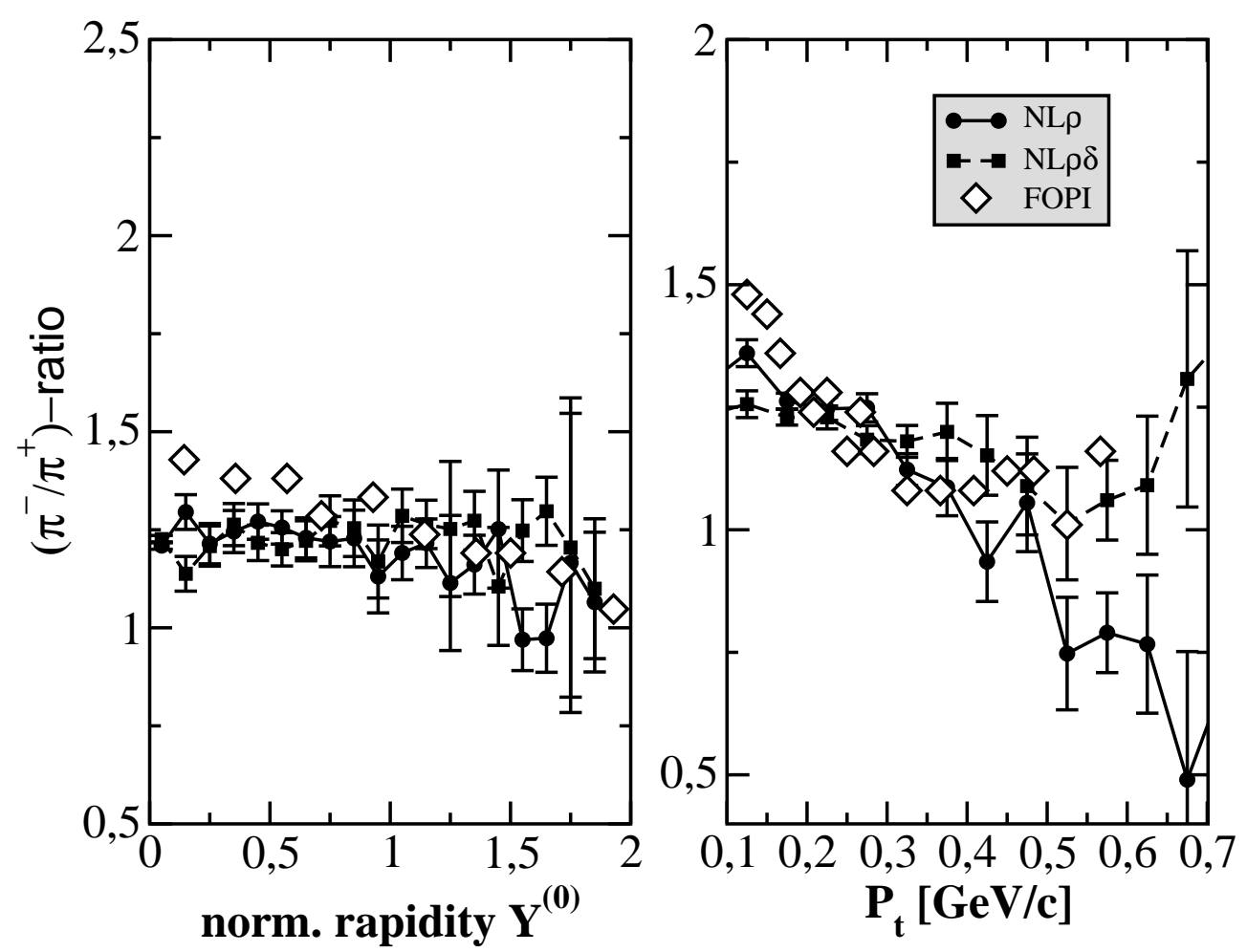

Fig. 12. $\pi^{-} / \pi^{+}$-ratio for central $R u+R u$-collisions as a function of rapidity (left) and transverse momentum (right) at mid-rapidity $\left(\left|Y^{(0)}\right| \leq 0.15\right)$. Same models as in Fig. 10. Data are taken from Ref. [50].

scalar field in the hadronic model. This seems in better agreement with the data, in particular for high- $P_{t}$ emitted pions.

We finally remark that this already clean indication could be further supported by studying instead of ${ }^{96} R u+{ }^{96} R u(N / Z=1.18)$ more asymmetric cases, like ${ }^{132} \mathrm{Sn}+{ }^{124} \mathrm{Sn}(N / Z=1.56)$ or ${ }^{197} \mathrm{Au}+{ }^{197} \mathrm{Au}(N / Z=1.49)$ colliding systems, and looking at similar more exclusive data.

\section{Summary and outlook}

In this paper we investigate in detail the density and momentum dependence of the symmetry energy in nuclear matter properties, finite nuclei and, in particular, in dynamical situations of intermediate energy heavy ion collisions. We discuss isospin effects in terms of the Lorentz structure of the isovector mean field. In addition to the usual vector-isovector $\rho$-meson we introduce a scalar, isovector $\delta$-like field, naturally keeping the same bulk asymmetry parameter at saturation. This will, however, change the high density and high momentum behavior of the symmetry term. We study the effects arising from the $\delta$-meson using different models of the Relativistic Mean Field $(R M F)$ theory, then stressing the role of Lorentz covariant properties of the isovector mean field in a general way. 
The comparisons between Non-Linear Walecka type ( $N L$, constant couplings) and the microscopically motivated density dependent hadron field $(D D H$, density dependent couplings) models show that the effects of the $\delta$ meson are of general nature and that they originate from the different covariant properties of the vector $\rho$ - and scalar $\delta$-fields at high baryon densities.

We have applied the models of asymmetric nuclear matter to finite nuclei and heavy ion collisions.

The studies of nuclear structure show that the effects of including the isovector $\delta$-channel are very small, except if one goes much beyond the valley of stability near the neutron drip line. Observable effects are found for very neutron-rich nuclei as an increased neutron diffusion at the nucleus surface and a larger binding energy per nucleon. Both results are due to the fact that in $n$-rich systems at low densities neutrons feel a larger attractive scalar self energy when including the $\delta$ meson. However, these effects are only moderate, in agreement with similar analyses of ref.[23], making it difficult to draw definite conclusions on the effective interactions. The main reason relies on the observation that in finite nuclei one explores the EOS around and below saturation density.

Thus, in order to investigate the Lorentz structure of the high density symmetry energy we have applied the models to dynamical situations in heavy ion collisions at intermediate energies, where relatively high baryon density regions can be reached during the reaction dynamics.

The isovector scalar and vector $\delta$ - and $\rho$-fields cancel each other in a similar way as for the isoscalar $\sigma$ - and $\omega$-fields. Thus, with the constraint to have the same symmetry energy at saturation, this cancellation is less effective at higher densities, with the repulsive $\rho$-meson gaining more importance $[15,16]$. With this pure relativistic mechanism the inclusion of a $\delta$-field will make the symmetry energy more repulsive at high baryon densities. Another very important transport effect of a scalar field in the isovector channel derives from the influence on the effective masses. In asymmetric matter we have now a splitting of the $n / p$ effective masses, also increasing with baryon density. This will affect nucleon velocity distributions and particle productions, particularly around the threshold. Finally, at high relativistic energies, due to the large velocity fields, one can expect large effects directly from the different covariant structure of the $\rho$ and $\delta$ mesons [25].

We have studied heavy ion collisions in a relativistic transport model of Boltzmann type and analyzed the results in terms of neutron and proton densities in space and time, particle production and pion flow observables. Within the same frame we have studied nucleon flows in a previous letter [25].

For $n$-rich colliding systems we find a strong decrease of the $n / p$-ratio in the bulk matter when including the $\delta$ meson in the isovector mean field. We in- 
terpret this effect by the joint action of a stiffer symmetry term and of a decrease of the neutron effective mass, more pronounced at high baryon densities, which both lead to more repulsion for neutrons than for protons. The influence of the $\delta$-meson in the dynamical situations has been mainly studied within the $N L$-models, where the differences in high density symmetry energy are actually not so strong. However, the $D D H$ models of density dependent coupling functions show very similar effects, even more pronounced due to the softer character of the symmetry energy. Moreover the dynamical implications of nucleon effective mass splitting are present in all cases. Therefore, the influence of the $\delta$-channel is a general feature arising from the differences in the Lorentz structure of the high density symmetry energy.

The relativistic form of the high density isovector EOS can also be directly seen in terms of particle production which can be experimentally measured. For this reason we have focussed our study on the influence of the $\delta$-meson on pion production. We evaluate from the reaction simulations the ratios of charged pions as function of time and space, energy, rapidity and transverse momentum. In general for all the colliding systems and energies we observe a reduction of the $\left(\pi^{-} / \pi^{+}\right)$-ratio in the models containing the $\delta$-meson.

This is due to the fact that the $\delta$ meson makes the high density region more proton-rich, see before. This induces a reduction in the formation of $\Delta^{0,-}$ resonances and thus in the production of negative charged pions. Moreover the lower neutron effective masses represent an efficient mechanism to directly quench the formation of negative $\Delta$-resonances. This is particularly evident at lower energies, around the threshold for resonance production.

The effect of the $\delta$-field as discussed above is enhanced in the momentum spectra, i.e. the rapidity and transverse momentum distributions, due to the interplay with the Coulomb interaction which affects differently the $\pi^{+}$and $\pi^{-}$distributions. Thus a clear, and likely detectable, effect of the $\delta$-field is predicted in the flow observables of the $\left(\pi^{-} / \pi^{+}\right)$-ratios, as well as in the incident energy dependence.

The comparison with the experiments seems to indicate that the introduction of the $\delta$ meson in the isovector mean field is important, i.e. as a way to promote, in $n$-rich systems, a larger vector repulsive field for high energy neutrons, joint to a smaller effective mass. We remark that both effects are mainly due to the relativistic dynamics and not necessarily directly related to the high density behavior of the symmetry energy.

However, in order to strengthen these conclusions one needs more precise experimental data, with more asymmetric, even unstable, ion beams, not only on pion production but simultanously on collective flow of neutrons and protons to study the so called isospin collective flow [25]. 
In the same heavy ion collisions, with high isospin density, the threshold production of other particles and resonances would also provide very relevant information.

At this level of investigation we conclude that relativistic studies of asymmetric static and dynamic nuclear matter appear very important in order to directly probe the Lorentz structure of the isovector part of the nuclear equation of state. 


\section{References}

[1] W. Reisdorf and H.G. Ritter, Annu. Rev. Nucl. Part. Sci. 47, 663 (1997);

N. Herrmann, J.P. Wessels, T. Wienold, Annu. Rev. Nucl. Part. Sci. 49, 581 (1999).

[2] B. D. Serot, J. D. Walecka, Adv. Nucl. Phys., 16, 1 (1986).

[3] R. Machleidt, Adv. Nucl. Phys., 19, 189 (1989).

[4] R. Brockmann and R. Machleidt, Phys. Rev. C42, 1965 (1990).

[5] R. Malfliet, Nucl. Phys. A545, 3c (1992).

[6] B. ter Haar, R. Malfliet, Phys. Rep. 149, 207 (1987) and Phys. Rev. C36, 1611 (1987);

T. Gross-Boelting, C. Fuchs, A. Faessler, Nucl. Phys. A648, 105 (1999).

[7] C.M. Ko, Prog. Part. Nucl. Phys. 42, 109 (1999) and refs. therein.

[8] W. Cassing and E.L. Bratkovskaya, Phys. Rep. 308, 65 (1999);

C. Sturm et al. (KaoS Collaboration), Phys. Rev. Lett. 86, 39 (2001).

[9] C. Fuchs et al., Phys. Rev. Lett. 86, 1974 (2001).

[10] H. Stöcker and W. Greiner, Phys. Rep. 137, 277 (1986);

P. Danielewicz, Roy A. Lacey, et al., Phys. Rev. Lett. 81, 2438 (1998);

C. Pinkenburg et al., Phys. Rev. Lett. 83, 1295 (1999).

[11] S. Typel and B.A. Brown, Phys. Rev. C64, 027302 (2001).

[12] R.J. Furnstahl, Nucl. Phys. A706, 85 (2002).

[13] V. Baran, M. Colonna, V. Greco, M. Di Toro, M. Zielinska Pfabe' and H.H. Wolter, Nucl.Phys. A706, 603 (2002).

[14] Bao-An Li, Phys. Rev. Lett. 85, 4221 (2000).

[15] B. Liu, V. Greco, V. Baran, M. Colonna and M.Di Toro, Phys. Rev. C65, 045201 (2002).

[16] V. Greco, M. Colonna, M. Di Toro and F. Matera, Phys. Rev. C67, 015203 (2003).

[17] R.J.Furnstahl and B.D.Serot, Comm. Nucl. Part. Phys. 2, A23 (2000) and refs. therein.

[18] R.J.Furnstahl, Next Generation Relativistic Models, arXiv:nucl-th/0307111.

[19] C. Fuchs, H. Lenske and H.H. Wolter, Phys. Rev. C52, 3043 (1995).

[20] F. Hofmann, C.M. Keil, H. Lenske, Phys.Rev. C64, 034314 (2001).

[21] E. Schiller and H. Muether, Eur.Phys.J. A11, 15 (2001). 
[22] Z. Ma and L. Liu, Phys.Rev. C66, 02434 (2002).

[23] S. Typel and H.H. Wolter, Nucl. Phys. A656, 331 (1999).

[24] Bao-An Li, Phys. Rev. C67, 017601 (2003) and refs. therein.

[25] V. Greco, V. Baran, M. Colonna, M. Di Toro, T. Gaitanos and H.H. Wolter, Phys. Lett. B562 215 (2003).

[26] Q. Li, Z. Li, Mod. Phys. Lett. A17, 375 (2002).

[27] L.W. Chen, C.M. Ko, Bao-An Li, Phys. Rev. C68, 017601 (2003).

[28] B. Blättel, V. Koch, U. Mosel, Rep. Prog. Phys. 56, 1 (1993).

[29] C. Fuchs et al., Nucl. Phys. A603, 471 (1996).

[30] We note that when the effective $\delta$-field is included we have a splitting of the neutron-proton effective masses in asymmetric matter. From Eq.7 we see that in n-rich systems the neutron effective mass is smaller with a splitting that is increasing with baryon density (for a constant $\delta$ coupling). We can expect interesting transport and threshold effects.

[31] P. Ring, Prog. Part. Nucl. Phys. 78, 193 (1996).

[32] G.A. Lalazissis, J. Konig and P. Ring, Phys. Rev. C55, 540 (1997).

[33] T. Niksic, D. Vtretenar and P. Ring, Phys. Rev. C66, 064302 (2002).

[34] F. de Jong, H. Lenske, Phys. Rev. C57, 3099 (1998).

[35] P. Danielewicz, Nucl. Phys. A673, 375 (2000).

[36] W. Botermans, R. Malfliet, Phys. Rep. 198, 115 (1990).

[37] C. Fuchs, H.H. Wolter, Nucl. Phys. A589, 732 (1995).

[38] C. Fuchs, T. Gaitanos, H.H. Wolter, Phys. Lett. B381, 23 (1996).

[39] G.F. Bertsch, S. Das Gupta, Phys. Rep. 160, 189 (1988).

[40] For the collision term we use here a modified version of the Tübingen $R Q M D$ simulation code, see [9] and refs. therein.

[41] J. Cugnon, D.L. Hote, J. Vandermeulen, Nucl. Instr. Meth. B111, 215 (1996).

[42] H. Huber, J. Aichelin, Nucl. Phys. A573, 587 (1994).

[43] A.B. Larionov et al., Nucl. Phys. A696, 747 (2001).

[44] Workshop on Transport Theories for Heavy Ion Collisions, 19-24.05.2003, ECT* Trento, Italy.

[45] L. Scalone, M. Colonna, M. Di Toro, Phys. Lett. B461, 9 (1999).

[46] W. Reisdorf (FOPI collaboration), private communication of very preliminary data. 
[47] A.Wagner, PhD-Thesis, 1996, TH-Darmstadt, Germany; C. Sturm (KAOS collaboration), private communication.

[48] V.S. Uma Maheswari et al., Phys. Rev. C57, 922 (1998).

[49] In our framework this is a characteristic relativistic effect that should be somewhat included in the corresponding non-relativistic models. The importance of this contribution was not realized so far in previous nonrelativistic studies [24], where no isospin effects were included in the momentum dependent part of the effective forces. In this case the differences in the used symmetry energy parametrizations at supra-normal densities were very pronounced and therefore they resulted in very large effects with respect to earlier neutron emission and particle production.

[50] B. Hong et al. (FOPI collaboration), GSI-Report 2002.

[51] T. Gaitanos, C. Fuchs, H. H. Wolter, A. Faessler, Eur. Phys. J. A12, 421 (2001). 